\title{
LOCAL TOPOLOGICAL OBSTRUCTION FOR DIVISORS
}

\author{
INDRANIL BISWAS AND ANANYO DAN
}

\begin{abstract}
Given a smooth, projective variety $X$ and an effective divisor $D \subseteq X$, it is wellknown that the (topological) obstruction to the deformation of the fundamental class of $D$ as a Hodge class, lies in $H^{2}\left(\mathcal{O}_{X}\right)$. In this article, we replace $H^{2}\left(\mathcal{O}_{X}\right)$ by $H_{D}^{2}\left(\mathcal{O}_{X}\right)$ and give an analogous topological obstruction theory. We compare the resulting local topological obstruction theory with the geometric obstruction theory (i.e., the obstruction to the deformation of $D$ as an effective Cartier divisor of a first order infinitesimal deformations of $X$ ). We apply this to study the jumping locus of families of linear systems and the Noether-Lefschetz locus. Finally, we give examples of first order deformations $X_{t}$ of $X$ for which the cohomology class $[D]$ deforms as a Hodge class but $D$ does not lift as an effective Cartier divisor of $X_{t}$.
\end{abstract}

\section{Contents}

1. Introduction 2

List of frequently used notations 3

2. Preliminaries 4

2.1. Cohomology with support 4

2.2. Local description of cohomology class of divisors 4

2.3. Inner multiplications with cohomology class of divisors 5

2.4. Relations with the cup product 6

2.5. Hodge locus for divisors 6

3. Torsion geometric obstruction $\quad 7$

3.1. Cokernel of the inner multiplication $\lrcorner\{D\}^{\prime} \quad 7$

3.2. Geometric obstruction 8

4. Local topological obstruction 9

4.1. Topological obstruction 10

4.2. Comparison with the geometric obstruction 12

5. Applications 14

5.1. Jumping locus of linear system 14

5.2. Noether-Lefschetz locus 16

6. An example 17

Acknowledgements $\quad 19$

$\begin{array}{ll}\text { References } & 19\end{array}$

2010 Mathematics Subject Classification. 14B10, 14B15, 14C30, 14C20, 14C25, 14D07.

Key words and phrases. Obstruction theories; Hodge locus; semi-regularity map; deformation of linear systems; Noether-Lefschetz locus. 


\section{INTRODUCTION}

The base field $k$ is always assumed to be algebraically closed of characteristic zero. Consider a family of smooth, projective varieties

$$
\pi: \mathcal{X} \longrightarrow B
$$

parameterized by a complex manifold $B$. Fix a closed point $o \in B$ and denote by $X:=\pi^{-1}(o)$ the fiber over $o$. Let $D \subseteq X$ be an effective divisor. Recall that, by Hodge decomposition, $H^{2}(X, \mathbb{C})$ decomposes as a direct sum of sub-vector spaces $H^{i, 2-i}(X)$ for $0 \leq i \leq 2$. The integral elements of $H^{1,1}(X)$, meaning the elements of $H^{1,1}(X) \cap\left(H^{2}(X, \mathbb{Z}) /(\right.$ torsion $)$ ), are known as Hodge classes. It is well-known that the fundamental class of a divisor, given by the first Chern class of the associated line bundle, is a Hodge class, which is proved using the exponential short exact sequence.

There is an obvious "topological" obstruction for an effective divisor $D \subseteq X$ to deform along with the variety $X$ : the fundamental class $[D]$ of the divisor $D$ is needed to deform as a Hodge class. Using the Lefschetz $(1,1)$-theorem, this topological obstruction is precisely the obstruction to the deformation of the invertible sheaf $\mathcal{O}_{X}(D)$. Using the exponential short exact sequence, one can then check that this obstruction actually lies in $H^{2}\left(X, \mathcal{O}_{X}\right)$. We will say that the fundamental class of $D$ deforms as a Hodge class along $t \in T_{o} B$ if the invertible sheaf $\mathcal{O}_{X}(D)$ deforms to an invertible sheaf on the first order infinitesimal deformation $X_{t}$ of $X$, in the direction of $t$. If the obstruction vanishes, we may further ask whether the divisor $D$ deforms as an effective Cartier divisor in $X_{t}$ ? This happens if and only if the section $s_{D}$ of $\mathcal{O}_{X}(D)$ cutting out the divisor $D$ deforms to a global section of the infinitesimal deformations of the invertible sheaf $\mathcal{O}_{X}(D)$. It is known that the obstruction to such deformations of the section $s_{D}$ lies in $H^{1}\left(X, \mathcal{O}_{X}(D)\right)$ (see $\left.[15, \S 3.3]\right)$. In this article, we replace the classical topological obstruction space $H^{2}\left(X, \mathcal{O}_{X}\right)$ by the local cohomology group $H_{D}^{2}\left(\mathcal{O}_{X}\right)$.

We prove the following (see Theorem 3.3):

Theorem 1.1. Consider deformations of $X$ parameterized by $B$ with base point $o \in B$, as above. To each effective divisor $D \subseteq X$ and tangent vector $t \in T_{o} B$, there is a canonically associated element $\xi_{D}^{t} \in H_{D}^{2}\left(\mathcal{O}_{X}\right)$ that satisfies the following condition: The vanishing of $\xi_{D}^{t}$ is equivalent to the existence of an integer $n>0$ such that $n D$ deforms as an effective Cartier divisor, as $X$ deforms in the direction of $t$.

Theorem 1.1 says that given an effective divisor $D$ in $X$, the obstruction to the deformation of a high enough multiple of $D$ as an effective Cartier divisor lies in $H_{D}^{2}\left(\mathcal{O}_{X}\right)$. Of course, if the obstruction vanishes then the fundamental class of $D$ deforms as a Hodge class. The question then arises, if no multiple of $D$ deforms as an effective Cartier divisor, is it still possible for the fundamental class of $D$ to deform as a Hodge class? As noted in Remark 4.1, this is possible. However, it can be shown that the difference between the two obstruction theories (i.e., the topological obstruction for the deformation of the fundamental class of $D$ as a Hodge class and the geometric obstruction for the deformation of a multiple of $D$ as an effective Cartier divisor) arises from 1-cocycles of the structure sheaf of an open subset $U$ of $X$ such that $X \backslash U$ is of codimension at least 2 in $X$. In particular, we prove (see Theorem 4.2):

Theorem 1.2. There exists a proper, closed subvariety $T \subseteq D$ (proper meaning the dimension of $T$ is strictly less than that of $D$ ) such that for the natural morphism

$$
\Phi_{(T \subseteq D)}: H^{1}\left(\mathcal{O}_{X \backslash T}\right) \longrightarrow H^{1}\left(\mathcal{O}_{X \backslash D}\right) \longrightarrow H_{D}^{2}\left(\mathcal{O}_{X}\right),
$$

and any tangent vector $t \in T_{o} B$, the fundamental class $[D]$ deforms as a Hodge class in the direction of $t$ if and only if the image of $\xi_{D}^{t} \in H_{D}^{2}\left(\mathcal{O}_{X}\right)$ defined in Theorem 1.1 above, maps to zero in $\operatorname{coker}\left(\Phi_{(T \subseteq D)}\right)$. 
In other words, the topological obstruction space is in fact contained in the cokernel of the morphism $\Phi_{(T \subseteq D)}$ mentioned above. The natural question to ask is, whether Theorem 1.2 holds for any proper, closed subvariety of the divisor $D$ ? Before we answer this question, we fix a couple of definitions. Given a proper, closed subvariety $T$ of $D$, we say that $D$ is $T$-semi-regular if Theorem 1.2 above holds for such a choice of $T$. We say that $D$ is effective Lefschetz if it satisfies the following property: for any $t \in T_{o} B$, the fundamental class $[D]$ deforms as a Hodge class along $t$ if and only if a multiple of $D$ deforms as an effective Cartier divisor, as $X$ deforms along $t$. In this context the following is proved (see Theorem 4.4):

Theorem 1.3. The divisor $D$ is $T$-semi-regular for every proper, closed subvariety $T$ of $D$ if and only if $D$ is effective Lefschetz.

We give an explicit example in Theorem 6.3 of a divisor which is not effective Lefschetz. See Remark 4.1 for a simpler example, given by the referee.

Let us now compare the above results with some classical results. We will call an effective divisor $D \subseteq X$ saturated if for every first order infinitesimal deformation of $X$, the divisor $D$ deforms as an effective Cartier divisor if and only if every positive multiple of $D$ deforms as an effective Cartier divisor. By Theorem 1.1, the divisor $D$ is saturated if and only if the natural morphism from the geometric obstruction space $\mathbf{O}_{D}^{\pi} \subseteq H^{1}\left(\mathcal{N}_{D \mid X}\right)$ (i.e., obstruction to the deformation of $D$ as an effective Cartier divisor) to $H_{D}^{2}\left(\mathcal{O}_{X}\right)$, induced by the natural morphism from $H^{1}\left(N_{D \mid X}\right)$ to $H_{D}^{2}\left(\mathcal{O}_{X}\right)$ (see $\S 2.4$ ), is injective (see Corollary 3.5). Note that, for saturated divisors, being effective Lefschetz is equivalent to the injectivity of the natural morphism from the geometric obstruction space $\mathbf{O}_{D}^{\pi}$ to the topological obstruction space $\operatorname{Obs}_{D}^{\pi} \subseteq H^{2}\left(\mathcal{O}_{X}\right)$ (i.e., the obstruction to deforming the fundamental class of $D$ as a Hodge class). Note that semi-regular sub-varieties obey such an injectivity statement. Recall that the theory of semi-regular subvarieties was first developed by Kodaira-Spencer [12] in the case of divisors, and subsequently it was generalized for the higher codimensions by Bloch [1]. However, semi-regularity is a rather rare phenomenon (see [5]). In contrast, saturated divisors are much more common than semiregular divisors. For example, a high enough multiple of any effective divisor is saturated.

Application: One can immediately apply these results to study the jumping locus of families of linear systems. Consider a flat family of smooth, projective varieties $\mathcal{X}$ parameterized by a complex manifold $B$. Fix a point $o \in B$. Let $\mathcal{L}$ be an invertible sheaf over $\mathcal{X}$. Denote by $W$ the universal sub-locus of $B$ containing the point $o$ such that for every $w \in W$, the dimension of the linear system associated to $\mathcal{L}_{w}:=\left.\mathcal{L}\right|_{\mathcal{X}_{w}}$ is constant and every sub-loci satisfying the same properties factor through $W$ (see $\S 5.1$ for a precise definition). We prove (see Theorem 5.3):

Theorem 1.4. Denote by $\mathcal{L}_{o}$ the restriction of $\mathcal{L}$ to the fiber over o. The tangent space at o to $W$ coincides with that of the parameterizing space $B$ (i.e., up to first order, the dimension of the linear system over the point $o \in B$ does not jump) if and only if every effective divisor $D$, in the linear system defined by $\mathcal{L}_{o}$, is $T$-semi-regular for every proper, closed subvariety $T \subseteq D$.

List of frequently used notations. We list a set of frequently used notations. Let $X$ be a smooth, projective variety and $D$ be an effective divisor in $X$. We then denote by:

(1) $\mathcal{H}_{D}^{i}(-)$ the local cohomology sheaf defined in $\S 2.1$.

(2) $\lrcorner\{D\}$ the contraction map from the tangent sheaf $\mathcal{T}_{X}$ to $\mathcal{H}_{D}^{1}\left(\mathcal{O}_{X}\right)$, defined in $\S 2.3$.

(3) $\lrcorner\{D\}^{\prime}$ the contraction map from the normal sheaf $\mathcal{N}_{D \mid X}$ to $\mathcal{H}_{D}^{1}\left(\mathcal{O}_{X}\right)$, defined in $\S 2.3$.

(4) $\pi_{D}$ is the morphism from $H^{1}\left(\mathcal{T}_{X}\right)$ to $H^{1}\left(\mathcal{H}_{D}^{1}\left(\mathcal{O}_{X}\right)\right) \cong H_{D}^{2}\left(\mathcal{O}_{X}\right)$ induced by $\lrcorner\{D\}$.

(5) $\pi_{D}^{\prime}$ is the morphism from $H^{1}\left(\mathcal{N}_{D \mid X}\right)$ to $H^{1}\left(\mathcal{H}_{D}^{1}\left(\mathcal{O}_{X}\right)\right) \cong H_{D}^{2}\left(\mathcal{O}_{X}\right)$ induced by $\lrcorner\{D\}^{\prime}$.

(6) $\Phi_{D}$ is the natural morphism from $H_{D}^{2}\left(\mathcal{O}_{X}\right)$ to $H^{2}\left(\mathcal{O}_{X}\right)$ guaranteed by Lemma 2.1 below.

(7) $\rho_{D}$ is the natural morphism from $\mathcal{T}_{X}$ to $\mathcal{N}_{D \mid X}$ defined in $\S 3.2$. 
(8) $\mathrm{Ob}_{D}$ from $H^{1}\left(\mathcal{T}_{X}\right)$ to $H^{1}\left(\mathcal{N}_{D \mid X}\right)$ is the natural obstruction map, induced by $\rho_{D}$.

(9) KS denotes the Kodaira-Spencer map (see $\S 2.5$ ).

(10) $\Phi_{(T \subseteq D)}$ for a proper closed subvariety $T$ of $D$ is the composition

$$
H^{1}\left(\mathcal{O}_{X \backslash T}\right) \rightarrow H^{1}\left(\mathcal{O}_{X \backslash D}\right) \rightarrow H_{D}^{2}\left(\mathcal{O}_{X}\right) .
$$

(11) $\eta_{T}$ is the natural morphism from the cokernel of $\Phi_{(T \subseteq D)}$ to $H^{2}\left(\mathcal{O}_{X}\right)$ defined in (4.2).

\section{Preliminaries}

In this section we recall the local description of an effective divisor $D$ contained in a smooth projective variety $X$. Using this, we define inner multiplications $\lrcorner\{D\}$ and $\lrcorner\{D\}^{\prime}$. We end the section with recollections of some basics on Hodge loci.

2.1. Cohomology with support. Let $X$ be a smooth projective variety and $D \subseteq X$ be an effective divisor. Let $\mathcal{F}$ be a sheaf of $\mathcal{O}_{X}$-modules. A (local) section $s$ of $\mathcal{F}$ is said to be supported over $D$ if $s$ is annihilated by a power of the ideal sheaf $\mathcal{I}_{D \mid X}$ of $D$ (as a subscheme of $X$ ). In particular, the sheaf $\underline{\Gamma}_{D}(\mathcal{F})$ of sections of $\mathcal{F}$ supported over $D$ is the sheaf associated to the presheaf which assigns to an open subset $U \subseteq X$, the $\mathcal{O}_{X}(U)$-module

$$
\left\{s \in \mathcal{F}(U) \mid \mathcal{I}_{D \mid X}(U)^{n} . s=0 \text { for some } n>0\right\} .
$$

Note that $\underline{\Gamma}_{D}(\mathcal{F})$ is isomorphic to $\underline{\Gamma}_{D_{\text {red }}}(\mathcal{F})$, where $D_{\text {red }}$ is the reduced scheme associated to $D$. Let $\Gamma_{D}(\mathcal{F})$ be the space of global sections of the sheaf $\Gamma_{D}(\mathcal{F})$. Denote by $\mathcal{H}_{D}^{i}(\mathcal{F})$ (respectively, $H_{D}^{i}(X, \mathcal{F})$ ) the right derived functor of the left exact functor $\underline{\Gamma}_{D}(-)$ (respectively, $\Gamma_{D}(-)$ ) applied to the sheaf $\mathcal{F}$. See $[3$, Chapter 1] or $[10, \S 1]$ for further details. We now recall the following useful result that encodes the difference between the cohomology groups $H^{i}(\mathcal{F})$ and $H_{D}^{i}(\mathcal{F})$ :

Lemma 2.1 ( [10, Corollary 1.1.9]). Let $\mathcal{F}$ be a quasi-coherent sheaf on $X$. Let $U:=X \backslash D$ be the complement with $j: U \hookrightarrow X$ the inclusion. There is a long exact sequence

$$
\begin{aligned}
0 \longrightarrow H_{D}^{0}(X, \mathcal{F}) \longrightarrow H^{0}(X, \mathcal{F}) \longrightarrow H^{0}\left(U,\left.\mathcal{F}\right|_{U}\right) \longrightarrow H_{D}^{1}(X, \mathcal{F}) \\
\longrightarrow H^{1}(X, \mathcal{F}) \longrightarrow H^{1}\left(U,\left.\mathcal{F}\right|_{U}\right) \longrightarrow H_{D}^{2}(X, \mathcal{F}) \longrightarrow \cdots .
\end{aligned}
$$

Similarly, there is a short exact sequence of sheaves

$$
0 \longrightarrow \mathcal{H}_{D}^{0}(X, \mathcal{F}) \longrightarrow \mathcal{H}^{0}(X, \mathcal{F}) \longrightarrow \mathcal{H}^{0}\left(U,\left.\mathcal{F}\right|_{U}\right) \longrightarrow \mathcal{H}_{D}^{1}(X, \mathcal{F}) \longrightarrow 0,
$$

and $\mathcal{H}_{D}^{i+1}(\mathcal{F}) \cong R^{i} j_{*}\left(\left.\mathcal{F}\right|_{U}\right)$ for all $i>0$.

2.2. Local description of cohomology class of divisors. Let $D \subseteq X$ be as in $\S 2.1$. Define $U:=X \backslash D$, and let $j: U \hookrightarrow X$ be the natural inclusion. Using Lemma 2.1, we have the short exact sequence

$$
0 \longrightarrow \Omega_{X}^{1} \longrightarrow j_{*} \Omega_{X \backslash D}^{1} \stackrel{\delta}{\longrightarrow} \mathcal{H}_{D}^{1}\left(\Omega_{X}^{1}\right) \longrightarrow 0 .
$$

Let $\left\{U_{i}\right\}_{i \in I}$ be an open affine covering of $X$ such that $D \cap U_{i}$ is defined by a single equation, say $f_{i}=0$ with $f_{i} \in \Gamma\left(U_{i}, \mathcal{O}_{X}\right)$. For $i \neq j$, since $f_{i}=\lambda_{i j} f_{j}$ over $U_{i} \cap U_{j}$ for some invertible regular section $\lambda_{i j}$ over $U_{i} \cap U_{j}$, we have by Leibniz rule,

$$
\delta\left(\frac{d f_{i}}{f_{i}}\right)=\delta\left(\frac{d f_{j}}{f_{j}}\right)+\delta\left(\frac{d \lambda_{i j}}{\lambda_{i j}}\right)
$$

over $U_{i} \cap U_{j}$. As $d \lambda_{i j} / \lambda_{i j} \in \Gamma\left(U_{i} \cap U_{j}, \Omega_{X}^{1}\right)$, (2.1) implies that the last term of the above equality vanishes. Hence, the collection of sections

$$
\left\{\delta\left(\frac{d f_{i}}{f_{i}}\right)\right\}_{i \in I}
$$


glue to give a global section, say $\{D\} \in H^{0}\left(\mathcal{H}_{D}^{1}\left(\Omega_{X}^{1}\right)\right)$. We now observe that in the case $\mathcal{F}$ is locally-free, then the cohomology group $H_{D}^{i}(\mathcal{F})$ is isomorphic to $H^{i-1}\left(\mathcal{H}_{D}^{1}(\mathcal{F})\right)$. In fact, we recall a more general result.

Proposition 2.2. Let $X$ be a scheme, and $Z$ be a local complete intersection subscheme in $X$. Let $\mathcal{F}$ be a sheaf of abelian groups on $X$. Then the spectral sequence with terms

$$
E_{2}^{p, q}=H^{p}\left(X, \mathcal{H}_{Z}^{q}(X, \mathcal{F})\right)
$$

converges to $H_{Z}^{p+q}(X, \mathcal{F})$. Furthermore, if $\mathcal{F}$ is a locally free $\mathcal{O}_{X}$-module, then

$$
H_{Z}^{p+q}(X, \mathcal{F}) \cong H^{p}\left(X, \mathcal{H}_{Z}^{q}(X, \mathcal{F})\right)
$$

where $q$ is the codimension of $Z$ in $X$ and $p \geq 0$.

Proof. The first statement is proven in [10, Proposition 1.4].

If $\mathcal{F}$ is locally free, then [4, Theorem 3.5.7] implies that $\mathcal{H}_{Z}^{k}(X, \mathcal{F})=0$ for $k \neq q$. Since

$$
E_{2}^{p, q}=H^{p}\left(X, \mathcal{H}_{Z}^{q}(X, \mathcal{F})\right) \Rightarrow H_{Z}^{p+q}(X, \mathcal{F}),
$$

we conclude that $H_{Z}^{p+q}(X, \mathcal{F}) \cong H^{p}\left(X, \mathcal{H}_{Z}^{q}(\mathcal{F})\right)$. This completes the proof.

In particular, Proposition 2.2 implies that $H^{0}\left(\mathcal{H}_{D}^{1}\left(\Omega_{X}^{1}\right)\right) \cong H_{D}^{1}\left(\Omega_{X}^{1}\right)$. By Lemma 2.1 , there exists a natural morphism from $H_{D}^{1}\left(\Omega_{X}^{1}\right)$ to $H^{1}\left(\Omega_{X}^{1}\right)$. The image of $\{D\}$ in $H^{1}\left(\Omega_{X}^{1}\right)$ under this morphism is the cohomology class $[D]$ of $D$ (see $[6]$ ).

2.3. Inner multiplications with cohomology class of divisors. Let $D \subseteq X$ be as in $\S 2.1$. Denote by $\mathcal{I}_{D \mid X}$ the ideal sheaf of $D$ in $X$ and $i: D \hookrightarrow X$ the natural inclusion. Recall,

$$
\mathcal{N}_{D \mid X} \cong \mathcal{H o m}_{X}\left(\mathcal{I}_{D \mid X}, i_{*} \mathcal{O}_{D}\right) \text { and } \mathcal{T}_{X} \cong \mathcal{H}_{\mathrm{om}}\left(\Omega_{X}^{1}, \mathcal{O}_{X}\right) \text {. }
$$

Fix an open affine covering $\left\{U_{i}\right\}_{i \in I}$ as in $\S 2.2$ such that for each $i \in I$, the intersection $D \cap U_{i}$ is defined by a regular section, say $f_{i} \in \Gamma\left(U_{i}, \mathcal{O}_{X}\right)$. On each $U_{i}$, a local section $\phi \in \Gamma\left(U_{i}, \mathcal{T}_{X}\right)$ (resp. $\left.\phi \in \Gamma\left(U_{i}, \mathcal{N}_{D \mid X}\right)\right)$ sends $d f_{i} \in \Gamma\left(U_{i}, \Omega_{X}^{1}\right)\left(\right.$ resp. $\left.f_{i} \in \Gamma\left(U_{i}, \mathcal{I}_{D \mid X}\right)\right)$ to $\phi\left(d f_{i}\right) \in \Gamma\left(U_{i}, \mathcal{O}_{X}\right)$ (resp. $\left.\phi\left(f_{i}\right) \in \Gamma\left(U_{i} \cap D, \mathcal{O}_{D}\right)\right)$. Using (2.1), we have the short exact sequence

$$
0 \longrightarrow \mathcal{O}_{X} \longrightarrow j_{*} \mathcal{O}_{X \backslash D} \stackrel{\delta^{\prime}}{\longrightarrow} \mathcal{H}_{D}^{1}\left(\mathcal{O}_{X}\right) \longrightarrow 0 \text {. }
$$

Define the contraction morphism

$$
\lrcorner\{D\}: \mathcal{T}_{X} \longrightarrow \mathcal{H}_{D}^{1}\left(\mathcal{O}_{X}\right) \quad(\text { respectively, }\lrcorner\{D\}^{\prime}: \mathcal{N}_{D \mid X} \longrightarrow \mathcal{H}_{D}^{1}\left(\mathcal{O}_{X}\right)\right),
$$

which on each $U_{i}$ takes $\phi \in \Gamma\left(U_{i}, \mathcal{T}_{X}\right)$ (respectively, $\left.\phi \in \Gamma\left(U_{i}, \mathcal{N}_{D \mid X}\right)\right)$ to

$$
\delta^{\prime}\left(\frac{\phi\left(d f_{i}\right)}{f_{i}}\right) \in \Gamma\left(U_{i}, \mathcal{H}_{D}^{1}\left(\mathcal{O}_{X}\right)\right) \quad\left(\text { respectively, } \delta^{\prime}\left(\frac{\widetilde{\phi\left(f_{i}\right)}}{f_{i}}\right) \in \Gamma\left(U_{i}, \mathcal{H}_{D}^{1}\left(\mathcal{O}_{X}\right)\right)\right),
$$

where $\widetilde{\phi\left(f_{i}\right)}$ is a preimage of $\phi\left(f_{i}\right) \in \mathcal{O}_{D}\left(U_{i} \cap D\right)$ under the natural surjective morphism

$$
\mathcal{O}_{X}\left(U_{i}\right) \longrightarrow \mathcal{O}_{D}\left(U_{i} \cap D\right) \text {. }
$$

Note that, any two pre-images of $\phi\left(f_{i}\right)$ differ by a section of $\mathcal{I}_{D \mid X}\left(U_{i}\right)$. Since $g / f_{i}$ is a regular section over $U_{i}$ for any section $g \in \mathcal{I}_{D \mid X}\left(U_{i}\right)$, (2.2) implies that $\delta^{\prime}\left(g / f_{i}\right)=0$. As a result, \lrcorner$\{D\}^{\prime}(\phi)$ does not depend on the choice of the pre-image. In other words, $\lrcorner\{D\}^{\prime}$ is well-defined. Similar argument shows that the morphisms $\lrcorner\{D\}$ and $\lrcorner\{D\}^{\prime}$ does not depend on the choice of the local defining equations of $D$.

The morphisms $\lrcorner\{D\}$ and $\lrcorner\{D\}^{\prime}$ induce on cohomology the morphisms

$$
\pi_{D}: H^{1}\left(\mathcal{T}_{X}\right) \longrightarrow H^{1}\left(\mathcal{H}_{D}^{1}\left(\mathcal{O}_{X}\right)\right) \cong H_{D}^{2}\left(\mathcal{O}_{X}\right) \text { and }
$$




$$
\pi_{D}^{\prime}: H^{1}\left(\mathcal{N}_{D \mid X}\right) \longrightarrow H^{1}\left(\mathcal{H}_{D}^{1}\left(\mathcal{O}_{X}\right)\right) \cong H_{D}^{2}\left(\mathcal{O}_{X}\right)
$$

where the isomorphisms follow from Proposition 2.2. As pointed out by the referee, the morphism $\pi_{D}^{\prime}$ may also be obtained by pairing $H^{1}\left(\mathcal{N}_{D \mid X}\right)$ with $H^{n-2}\left(\left.K_{X}\right|_{D}\right)$ and then applying formal duality. More precisely, by the adjunction formula (see [8, Theorem III.7.11]), we have $\left.\mathcal{N}_{D \mid X}^{\vee} \otimes K_{D} \cong K_{X}\right|_{D}$ (here $K_{D}$ denotes the dualizing sheaf of $D$ ). By Serre duality, this implies

$$
H^{1}\left(\mathcal{N}_{D / X}\right)^{\vee} \cong H^{n-2}\left(\mathcal{N}_{D / X}^{\vee} \otimes K_{D}\right) \cong H^{n-2}\left(\left.K_{X}\right|_{D}\right)
$$

By formal duality (see [7, p. 48, Proposition 5.2]), we have an isomorphism of (not necessarily finite-dimensional) $\mathbb{C}$-vector spaces:

$$
{\underset{\varliminf}{m}}_{\lim } H^{n-2}\left(K_{X} \otimes \mathcal{O}_{X} / \mathcal{I}_{D \mid X}^{m}\right) \cong H_{D}^{2}\left(\mathcal{O}_{X}\right)^{\vee}
$$

where $\mathcal{I}_{D \mid X}$ is the ideal sheaf of $D$ in $X$. By the definition of inverse limit, this gives us a natural morphism

$$
H_{D}^{2}\left(\mathcal{O}_{X}\right)^{\vee} \cong{\underset{m}{m}}_{\lim } H^{n-2}\left(K_{X} \otimes \mathcal{O}_{X} / \mathcal{I}_{D \mid X}^{m}\right) \longrightarrow H^{n-2}\left(\left.K_{X}\right|_{D}\right) .
$$

As $H^{n-2}\left(\left.K_{X}\right|_{D}\right)$ is isomorphic to $H^{1}\left(\mathcal{N}_{D \mid X}\right)^{\vee}$, dualizing the resulting morphism from $H_{D}^{2}\left(\mathcal{O}_{X}\right)^{\vee}$ to $H^{1}\left(\mathcal{N}_{D \mid X}\right)^{\vee}$ will give us the morphism $\pi_{D}^{\prime}$ above.

2.4. Relations with the cup product. Let $X$ be a smooth, projective variety. Given two $\mathcal{O}_{X}$-modules $\mathcal{F}$ and $\mathcal{G}$, there is a natural cup-product morphism

$$
H^{p}(\mathcal{F}) \otimes H^{q}(\mathcal{G}) \longrightarrow H^{p+q}(\mathcal{F} \otimes \mathcal{G})
$$

See $[17, \S 5.3 .2]$ for a detailed description of this morphism. In the case when $\mathcal{G}$ is the dual of $\mathcal{F}$, we can further compose this morphism by the natural pairing from $\mathcal{F} \otimes \mathcal{F}^{\vee}$ to $\mathcal{O}_{X}$. More-precisely, we get a composed morphism

$$
H^{p}(\mathcal{F}) \otimes H^{q}\left(\mathcal{F}^{\vee}\right) \longrightarrow H^{p+q}\left(\mathcal{F} \otimes \mathcal{F}^{\vee}\right) \longrightarrow H^{p+q}\left(\mathcal{O}_{X}\right) .
$$

In the case, where $\mathcal{F}$ is the tangent sheaf $\mathcal{T}_{X}$, we have the morphism

$$
\bigcup: H^{1}\left(\mathcal{T}_{X}\right) \otimes H^{1}\left(\Omega_{X}^{1}\right) \longrightarrow H^{2}\left(\mathcal{O}_{X}\right)
$$

We now observe that for a given effective divisor $D$ in $X$, the induced morphism $\bigcup(-\otimes[D])$, sometimes denoted by $-\bigcup[D]$, can be expressed in terms of the contraction morphism $\lrcorner\{D\}$ described in $\S 2.3$ above. More precisely, using [1, Proposition 6.2], we have for any $t \in H^{1}\left(\mathcal{T}_{X}\right)$ that $t \bigcup[D]$ coincides with the image of $t$ under the composition

$$
H^{1}\left(\mathcal{T}_{X}\right) \stackrel{\pi_{D}}{\longrightarrow} H_{D}^{2}\left(\mathcal{O}_{X}\right) \stackrel{\Phi_{D}}{\longrightarrow} H^{2}\left(\mathcal{O}_{X}\right)
$$

where the morphism $\pi_{D}$ is induced by $\lrcorner\{D\}$ (see $\S 2.3$ ) and $\Phi_{D}$ follows from Lemma 2.1.

2.5. Hodge locus for divisors. Consider a family

$$
\pi: \mathcal{X} \longrightarrow B
$$

of smooth, projective varieties with a reference point $o \in B$. Let $X:=\pi^{-1}(o)$ be the inverse image. The differential of $\pi$ produces a short exact sequence of sheaves

$$
\left.0 \longrightarrow \mathcal{T}_{X} \longrightarrow \mathcal{T}_{\mathcal{X}}\right|_{X} \longrightarrow \pi^{*} T_{o} B \longrightarrow 0
$$

where $T_{o} B$ is the tangent space to $B$ at $o$. The Kodaira-Spencer map

$$
\mathrm{KS}: T_{o} B \cong H^{0}\left(\mathcal{X}, \pi^{*}\left(T_{o} B\right)\right) \longrightarrow H^{1}\left(\mathcal{X}, \mathcal{T}_{X}\right)
$$

is the coboundary morphism in the long exact sequence of cohomologies associated to the short exact sequence in (2.3). 
Let $D$ be an effective divisor in $X$. The Hodge locus associated to the cohomology class $[D]$, denoted $\mathrm{NL}([D])$, is the subspace of $B$ consisting of points $b \in B$ such that $[D]$ deforms to a Hodge class on $\mathcal{X}_{b}=\pi^{-1}(b)$. By the Lefschetz $(1,1)$-theorem, every cohomology class of a divisor arises as the first Chern class of a line bundle. As a result deformations of the cohomology class $[D]$ is equivalent to the deformation of the line bundle $\mathcal{O}_{X}(D)$. Hence, the subspace $\mathrm{NL}([D])$ can also be seen as a subspace of $B$ over which the invertible sheaf corresponding to $D$ deforms as an invertible sheaf. See $[18, \S 5.3 .1]$ for a scheme-theoretic description of $\mathrm{NL}([D])$.

Denote by $T_{o} \mathrm{NL}([D])$ the tangent space at $o$ of $\mathrm{NL}([D])$. By $[17$, Theorem 10.21], the tangent space $T_{o} \mathrm{NL}([D])$ to the Hodge locus $\mathrm{NL}([D])$ at the point $o$ consists of all $t \in T_{o} B$ such that $[D] \cup \operatorname{KS}(t)=0$. Given any $t \in T_{o} B$, we say that $[D]$ deforms as a Hodge class of $X_{t}$ if

$$
[D] \bigcup \mathrm{KS}(t)=0,
$$

where $X_{t}$ denotes the first order infinitesimal deformation of $X$ in the direction of $t$.

\section{TORSION GEOMETRIC OBSTRUCTION}

Let $X$ be a smooth, projective variety and $D \subseteq X$ be an effective divisor. In this section, we associate to a first order infinitesimal deformation $X_{t}$ of $X$ an element of $H_{D}^{2}\left(\mathcal{O}_{X}\right)$, the vanishing of which is equivalent to the deformation of a multiple of $D$ as an effective Cartier divisor of $X_{t}$ (Theorem 3.3).

3.1. Cokernel of the inner multiplication $\lrcorner\{D\}^{\prime}$. Note that, there is a natural morphism from $\mathcal{O}_{X}$ to $\mathcal{O}_{X}(D)$ defined by multiplication by a global section, say $s_{D} \in H^{0}\left(\mathcal{O}_{X}(D)\right)$. Then, for any $n>0$, the natural morphism from $\mathcal{O}_{X}$ to $\mathcal{O}_{X}(n D)$ is defined by multiplication by $s_{D}^{n}$. For simplicity of notation, we denote by

$$
s_{D}^{n}: \mathcal{H}_{D}^{1}\left(\mathcal{O}_{X}\right) \longrightarrow \mathcal{H}_{D}^{1}\left(\mathcal{O}_{X}(n D)\right)
$$

the morphism induced by multiplication by $s_{D}^{n}$. We then observe that the morphism $s_{D}^{n}$ is surjective with kernel isomorphic to $\mathcal{N}_{n D \mid X}$ :

Lemma 3.1. Let $X$ be a smooth projective variety, and let $D \subseteq X$ be an effective divisor. For every $n \geq 1$, there is a short exact sequence of the form

$$
0 \longrightarrow \mathcal{N}_{n D \mid X} \stackrel{\lrcorner\{n D\}^{\prime}}{\longrightarrow} \mathcal{H}_{D}^{1}\left(\mathcal{O}_{X}\right) \stackrel{s_{D}^{n}}{\longrightarrow} \mathcal{H}_{D}^{1}\left(\mathcal{O}_{X}(n D)\right) \longrightarrow 0,
$$

where $\lrcorner\{n D\}^{\prime}$ is the inner multiplication described in $\S 2.3$.

Proof. Let $j: X \backslash D \longrightarrow X$ be the natural inclusion. Consider the commutative diagram of short exact sequences

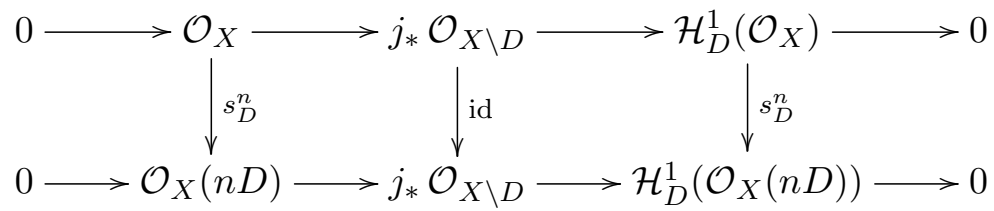

where the first horizontal exact sequence is the one in (2.2) and the second one is obtained after replacing $\mathcal{O}_{X}$ in $(2.2)$ by $\mathcal{O}_{X}(n D)$ (use the identification $\left.\left.\mathcal{O}_{X \backslash D} \cong \mathcal{O}_{X}(n D)\right|_{X \backslash D}\right)$. Let $\left\{U_{i}\right\}_{i \in I}$ be an affine open covering of $X$ such that for each $i \in I$, the intersection $D \cap U_{i}$ is defined by exactly one equation, say $f_{i} \in \Gamma\left(U_{i}, \mathcal{O}_{X}\right)$; the equation is unique up to multiplication by units. Consider now the short exact sequence

$$
0 \longrightarrow \mathcal{O}_{X} \stackrel{s_{D}^{n}}{\longrightarrow} \mathcal{O}_{X}(n D) \longrightarrow \mathcal{N}_{n D \mid X} \longrightarrow 0,
$$


where the morphism from $\mathcal{O}_{X}(n D)$ to $\mathcal{N}_{n D \mid X}$ is defined over each open set $U_{i}$ by sending any $g \in \Gamma\left(U_{i}, \mathcal{O}_{X}(n D)\right)$ to the morphism $\phi \in \operatorname{Hom}_{U_{i}}\left(\left.\mathcal{O}_{X}(-n D)\right|_{U_{i}}, \mathcal{O}_{n D \cap U_{i}}\right)$ satisfying the condition

$$
\phi\left(f_{i}^{n}\right)=\left.g f_{i}^{n} \quad \bmod \mathcal{O}_{X}(-n D)\right|_{U_{i}} .
$$

Using the Snake lemma and diagram chase applied to (3.2), we then get the short exact sequence

$$
0 \longrightarrow \mathcal{N}_{n D \mid X} \stackrel{\lrcorner\{n D\}^{\prime}}{\longrightarrow} \mathcal{H}_{D}^{1}\left(\mathcal{O}_{X}\right) \stackrel{s_{D}^{n}}{\longrightarrow} \mathcal{H}_{D}^{1}\left(\mathcal{O}_{X}(n D)\right) \longrightarrow 0 .
$$

This proves the lemma.

3.2. Geometric obstruction. Let $\pi: \mathcal{X} \longrightarrow B$ be a flat family of smooth projective varieties with a fixed base point $o \in B$. Let $\mathcal{X}_{b}:=\pi^{-1}(b)$ be the inverse image of any $b \in B$, and set $X:=\pi^{-1}(o)$.

Notation 3.2. Let $D \subseteq X$ be an effective divisor. There is a natural morphism

$$
\rho_{D}: \mathcal{T}_{X} \longrightarrow \mathcal{N}_{D \mid X}
$$

obtained as a composition of the restriction morphism from $\mathcal{T}_{X}$ to $\left.\mathcal{T}_{X}\right|_{D}$ with the morphism from $\left.\mathcal{T}_{X}\right|_{D}$ to $\mathcal{N}_{D \mid X}$ arising as the dual of the restriction to $D$ of the morphism

$$
\mathcal{O}_{X}(-D) \stackrel{s_{D}}{\longrightarrow} \mathcal{O}_{X} \stackrel{d}{\longrightarrow} \Omega_{X}^{1},
$$

where $s_{D}$ is the global section of $\mathcal{O}_{X}(D)$ corresponding to the divisor $D$. Let

$$
\mathrm{Ob}_{D}: H^{1}\left(\mathcal{T}_{X}\right) \longrightarrow H^{1}\left(\mathcal{N}_{D \mid X}\right)
$$

be the homomorphism of cohomology groups induced by $\rho_{D}$.

Recall, the morphism $\pi_{D}$ from $H^{1}\left(\mathcal{T}_{X}\right)$ to $H_{D}^{2}\left(\mathcal{O}_{X}\right)$ obtained by applying the cohomology functor to the contraction morphism $\lrcorner\{D\}$ from $\mathcal{T}_{X}$ to $\mathcal{H}_{D}^{1}\left(\mathcal{O}_{X}\right)$ described in $\S 2.3$. We now observe that the image of $\pi_{D}$ contains the obstruction to the deformation of a multiple of $D$.

Theorem 3.3. Let $t \in T_{o} B$ and $D \subseteq X$ an effective divisor. Then, $\pi_{D} \circ \mathrm{KS}(t)=0$ if and only if there exists an integer $n>0$ such that $n D$ deforms as an effective Cartier divisor in the first order infinitesimal deformation $X_{t}$ of $X$ corresponding to $t$.

Proof. Take any $t \in T_{o} B$. Denote by $X_{t}$ the first order infinitesimal deformation of $X$ corresponding to $t$. Recall, for any positive integer $n>0$, the effective divisor $n D$ deforms as an effective Cartier divisor in $X_{t}$ if and only if $\mathrm{Ob}_{n D} \circ \mathrm{KS}(t)$ vanishes (see [9, Theorem 6.2]). Therefore, to prove the theorem, we need to compare the obstruction morphisms $\mathrm{Ob}_{D}$ and $\mathrm{Ob}_{n D}$, where $n$ is some positive integer. Let $s_{D}$ be the global section of $\mathcal{O}_{X}(D)$ corresponding to the divisor $D$. Now, for any regular local section $f$ of $\mathcal{O}_{X}$, we have

$$
d\left(f s_{D}^{n}\right)=n s_{D}^{n-1} f d\left(s_{D}\right)+s_{D}^{n} d f .
$$

Restricting to $n D$, the last term of the equality vanishes. We then get the commutative diagram:

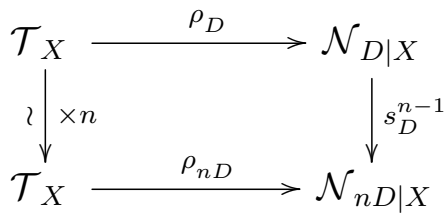


where the morphism from $\mathcal{N}_{D \mid X}$ to $\mathcal{N}_{n D \mid X}$ is induced by multiplication by $s_{D}^{n-1}$. This relates $\mathrm{Ob}_{D}$ to $\mathrm{Ob}_{n D}$. Now, by Lemma 3.1 we have the following diagram of short exact sequences:

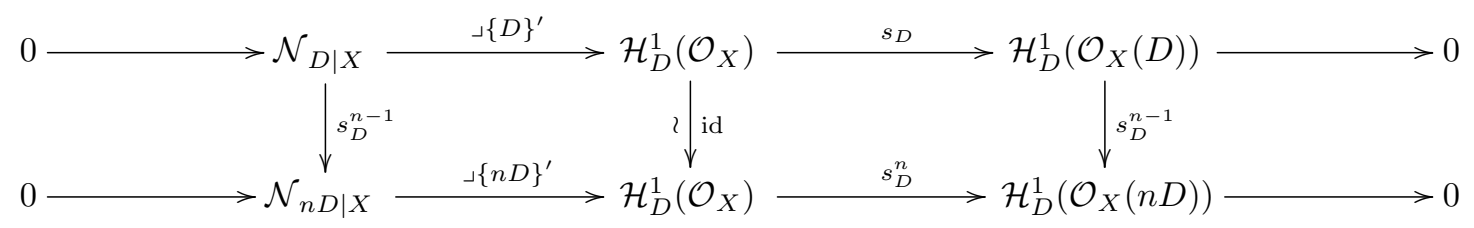

Note that, the contraction morphism $\lrcorner\{D\}$ from $\mathcal{T}_{X}$ to $\mathcal{H}_{D}^{1}\left(\mathcal{O}_{X}\right)$ factors as

$$
\mathcal{T}_{X} \stackrel{\rho_{D}}{\longrightarrow} \mathcal{N}_{D \mid X} \stackrel{\lrcorner\{D\}^{\prime}}{\longrightarrow} \mathcal{H}_{D}^{1}\left(\mathcal{O}_{X}\right) .
$$

This implies $\pi_{D}^{\prime} \circ \mathrm{Ob}_{D}=\pi_{D}$. The long exact sequence associated to (3.4) then implies that $\pi_{D} \circ \mathrm{KS}(t)=0$ if and only if there exists $f \in H^{0}\left(\mathcal{H}_{D}^{1}\left(\mathcal{O}_{X}(D)\right)\right)$ such that the image of $f$ under the coboundary map

$$
H^{0}\left(\mathcal{H}_{D}^{1}\left(\mathcal{O}_{X}(D)\right)\right) \longrightarrow H^{1}\left(\mathcal{N}_{D \mid X}\right)
$$

equals $\mathrm{Ob}_{D} \circ \mathrm{KS}(t)$. Observe that given any global section $f$ of $\mathcal{H}_{D}^{1}\left(\mathcal{O}_{X}(D)\right)$ there exists an integer $N>0$ such that for all $n \geq N, f s_{D}^{n-1}$ extends to a regular section of $\mathcal{O}_{X}(n D)$, hence defines a zero section in $H^{0}\left(\mathcal{H}_{D}^{1}\left(\mathcal{O}_{X}(n D)\right)\right)$ by the exact sequence given in Lemma 2.1. The theorem then follows by a simple diagram chase of the diagram of long exact sequences arising from (3.4). This proves the theorem.

Theorem 3.3 motivates the following definition.

Definition 3.4. For any $t \in T_{o} B$, denote by $X_{t}$ the first order infinitesimal deformation of $X$ corresponding to $t$. Let $D \subseteq X$ be an effective divisor. We say that $D$ is saturated, along $B$, if for every $t \in T_{o} B$, the divisor $D$ lifts as an effective Cartier divisor in $X_{t}$ if and only if $n D$ lifts as an effective Cartier divisor in $X_{t}$ for every $n \geq 1$.

Corollary 3.5. Let $D \subseteq X$ be an effective divisor. Then $D$ is saturated if and only if

$$
\operatorname{Im}\left(\mathrm{Ob}_{D} \circ \mathrm{KS}\right) \cap \operatorname{ker}\left(\pi_{D}^{\prime}: H^{1}\left(\mathcal{N}_{D \mid X}\right) \longrightarrow H^{1}\left(\mathcal{H}_{D}^{1}\left(\mathcal{O}_{X}\right)\right)\right)=0 .
$$

Proof. Using Theorem 3.3, note that the intersection of $\operatorname{Im}\left(\mathrm{Ob}_{D} \circ \mathrm{KS}\right)$ with $\operatorname{ker}\left(\pi_{D}^{\prime}\right)$ consists of all elements of the form $\mathrm{Ob}_{D} \circ \mathrm{KS}(t)$ such that there exists $n>0$ for which $n D$ deforms to an effective Cartier divisor in the first order infinitesimal deformation $X_{t}$ of $X$ corresponding to $t$, but $D$ does not deform. Hence, the divisor $D$ is saturated if and only if

$$
\operatorname{Im}\left(\mathrm{Ob}_{D} \circ \mathrm{KS}\right) \cap \operatorname{ker}\left(\pi_{D}^{\prime}\right)=0 .
$$

This proves the corollary.

\section{LOCAL TOPOLOGICAL OBSTRUCTION}

Throughout this section, $\pi: \mathcal{X} \longrightarrow B$ stands for a flat family of smooth projective varieties with a fixed base point $o \in B$. Let $\mathcal{X}_{b}:=\pi^{-1}(b)$ be the inverse image of any $b \in B$, and set $X:=\pi^{-1}(o)$. Let $D \subseteq X$ be an effective Cartier divisor. As discussed in the introduction, the topological obstruction to deforming the fundamental class of $D$ as a Hodge class, lie in $H^{2}\left(\mathcal{O}_{X}\right)$. The goal of this section is to replace $H^{2}\left(\mathcal{O}_{X}\right)$ by $H_{D}^{2}\left(\mathcal{O}_{X}\right)$. For this we consider the natural morphism $\Phi_{D}$ from $H_{D}^{2}\left(\mathcal{O}_{X}\right)$ to $H^{2}\left(\mathcal{O}_{X}\right)$. We observed in Theorem 3.3 that for any $t \in T_{o} B$, the element $\pi_{D} \circ \mathrm{KS}(t)$ measures the obstruction to deforming a multiple of $D$ as an effective Cartier divisor. It is of course possible for $\pi_{D} \circ \mathrm{KS}(t)$ to be non-zero, but $\Phi_{D} \circ \pi_{D} \circ \mathrm{KS}(t)$ to vanish (see Remark 4.1). However, we observe that all such $\pi_{D} \circ \mathrm{KS}(t)$ come from the 1-cocycle of the structure sheaf of the complement of $X$ by a codimension 2 subvariety $T$ (see Theorem 4.2). We study this further in Theorem 4.4 and Corollary 4.5. 
4.1. Topological obstruction. Let $D \subseteq X$ be an effective divisor. Recall the morphism $\pi_{D}$ from $H^{1}\left(\mathcal{T}_{X}\right)$ to $H_{D}^{2}\left(\mathcal{O}_{X}\right)$ defined in $\S 2.3$. Let

$$
\operatorname{Obs}_{D, \text { loc }}^{\pi}:=\operatorname{Im}\left(\pi_{D} \circ \mathrm{KS}\right) \subseteq H_{D}^{2}\left(\mathcal{O}_{X}\right) \text { and } \operatorname{Obs}_{D}^{\pi}:=\operatorname{Im}\left(\Phi_{D} \circ \pi_{D} \circ \mathrm{KS}\right) \subseteq H^{2}\left(\mathcal{O}_{X}\right)
$$

be the topological obstruction spaces associated to $D$, where $\Phi_{D}$ is the natural morphism from $H_{D}^{2}\left(\mathcal{O}_{X}\right)$ to $H^{2}\left(\mathcal{O}_{X}\right)$. It follows immediately that there exists a natural surjective morphism from $\operatorname{Obs}_{D \text {,loc }}^{\pi}$ to $\operatorname{Obs}_{D}^{\pi}$, induced by the morphism $\Phi_{D}$. It is possible that this morphism is not injective. In fact, we now give an example when this map is the zero map.

Remark 4.1. The example arises from the blow-up of two points, say $p, q$ in $\mathbb{P}^{2}$, as $p$ approaches $q$. More precisely, set $Y:=\mathbb{P}^{2} \times \mathbb{A}^{1}$ and take $T_{1} \subseteq Y$ (respectively, $T_{2} \subseteq Y$ ) closed subvarieties consisting of points of the form $([1: 0: t], t)$ (respectively, $([1: t: 0], t))$ as $t$ varies over $\mathbb{A}^{1}$. Let $Y_{1}$ be the blow-up of $Y$ along the closed subscheme $T_{1}$, and let $E_{1}$ be the associated exceptional divisor. Let $T_{2}^{\prime}$ be the strict transform of $T_{2}$ in $Y_{1}$. Denote by

$$
p: Y_{2} \rightarrow Y_{1}
$$

the blow-up of $Y_{1}$ along $T_{2}^{\prime}$, and let $E_{2} \subseteq Y_{2}$ be the exceptional divisor. Let $Y_{2,0}$ be the fiber over $0 \in \mathbb{A}^{1}$ of the natural morphism

$$
\pi: Y_{2} \longrightarrow \mathbb{A}^{1} \text {. }
$$

Note that the generic fiber of $\pi$ is the blow-up of $\mathbb{P}^{2}$ at two distinct points and in the limit the central fiber has an $A_{2}$-configuration of $\mathbb{P}^{1}$ s. Let, $E_{1,0}$ and $E_{2,0}$ respectively are the fibers over $0 \in \mathbb{A}^{1}$ of the natural morphisms $E_{1} \longrightarrow \mathbb{A}^{1}$ and $E_{2} \longrightarrow \mathbb{A}^{1}$. Note that, $E_{1,0}$ is isomorphic to $\mathbb{P}^{1}$. Denote by $D$ the strict transform of $E_{1,0}$ in $Y_{2}$. Clearly, $D$ is isomorphic to $\mathbb{P}^{1}$. Using [8, Propositions V.3.2 and V.3.6], we conclude that

$$
D^{2}+1=\left(D+E_{2,0}\right) \cdot D=p^{*}\left(E_{1,0}\right) \cdot D=\left(E_{1,0}\right)^{2}=-1 .
$$

Hence, $D^{2}=-2$. This implies $\mathcal{N}_{D \mid Y_{2,0}} \cong \mathcal{O}_{D}(-2)$. As $K_{D} \cong \mathcal{O}_{D}(-2)$, we have by Serre duality

$$
H^{1}\left(\mathcal{N}_{D \mid Y_{2,0}}\right) \cong H^{0}\left(\mathcal{O}_{D}\right)^{\vee} \cong \mathbb{C} \text {. }
$$

By [8, Proposition V.3.4], we have $H^{2}\left(\mathcal{O}_{Y_{2,0}}\right)=0$. This means that $\operatorname{Obs}_{D}^{\pi}=0$.

We will now observe that $\operatorname{Obs}_{D \text {,loc }}^{\pi}=\mathbb{C}$. For this purpose, we will show that $D$ or any positive multiple of $D$ does not deform even upto first order, as an effective Cartier divisor, as $Y_{2,0}$ deforms along $\mathbb{A}^{1}$ in the family $\pi$. Using Theorem 3.3, this will imply $\operatorname{Obs}_{D \text {,loc }}^{\pi} \subseteq H_{D}^{2}\left(\mathcal{O}_{X}\right)$ is non-trivial. As $H^{1}\left(\mathcal{N}_{D \mid Y_{2,0}}\right)=\mathbb{C}$, it will follow from definition that $\operatorname{Obs}_{D \text {,loc }}^{\pi}=\mathbb{C}$. We now prove the obstruction to the first-order infinitesimal deformation of $n D$ for any $n>0$. As $Y_{2,0}$ is a fiber over $0 \in \mathbb{A}^{1}$, we have $\mathcal{N}_{Y_{2,0} \mid Y} \cong \mathcal{O}_{Y_{2,0}}$. Then, the normal short exact sequence is of the form:

$$
0 \longrightarrow \mathcal{N}_{D \mid Y_{2,0}} \longrightarrow \mathcal{N}_{D \mid Y_{2}} \longrightarrow \mathcal{O}_{D} \longrightarrow 0
$$

Since $\mathcal{N}_{D \mid Y_{2,0}} \cong \mathcal{O}_{D}(-2)$, only one of the following is possible:

- Either $\mathcal{N}_{D \mid Y_{2}}$ is isomorphic to $\mathcal{O}_{D}(-2) \oplus \mathcal{O}_{D}$, in which case (4.1) splits,

- or $\mathcal{N}_{D \mid Y_{2}}$ is isomorphic to $\mathcal{O}_{D}(-1) \oplus \mathcal{O}_{D}(-1)$.

Note that, $D$ can be contracted to a 3 -fold with ordinary double point given by the blow-up of $Y$ along $T_{1} \cup T_{2}$. This implies that $N_{D \mid Y_{2}}$ must be isomorphic to $\mathcal{O}_{D}(-1) \oplus \mathcal{O}_{D}(-1)$ (see [14, p. $158,159]$ ). The obstruction to the first order deformation of $D$ (as an effective Cartier divisor) as $Y_{2,0}$ deforms along $\mathbb{A}^{1}$, is the image of $1 \in H^{0}\left(\mathcal{O}_{D}\right)$ under the coboundary map:

$$
H^{0}\left(\mathcal{O}_{D}\right) \longrightarrow H^{1}\left(\mathcal{N}_{D \mid Y_{2,0}}\right)
$$

arising from (4.1). Since $H^{0}\left(\mathcal{O}_{D}(-1) \oplus \mathcal{O}_{D}(-1)\right)=0$, the coboundary map is injective. Hence, the obstruction does not vanish. In other words, $D$ does not deform to first order as an effective 
Cartier divisor, as $Y_{2,0}$ deforms along $\mathbb{A}^{1}$. We now prove the same for higher multiples of $D$. Since $D^{2}=-2$, we have $H^{0}\left(\mathcal{O}_{D} \otimes \mathcal{O}_{Y_{2,0}}(m D)\right)=0$ for all $m>0$. Then for any $m>0$, the long exact sequence associated to short exact sequence:

$$
0 \longrightarrow \mathcal{O}_{Y_{2,0}}((m-1) D) \longrightarrow \mathcal{O}_{Y_{2,0}}(m D) \longrightarrow \mathcal{O}_{D} \otimes \mathcal{O}_{Y_{2,0}}(m D) \longrightarrow 0
$$

implies that

$$
H^{0}\left(\mathcal{O}_{Y_{2,0}}((m-1) D)\right)=H^{0}\left(\mathcal{O}_{Y_{2,0}}(m D)\right)
$$

and the morphism from $H^{1}\left(\mathcal{O}_{Y_{2,0}}((m-1) D)\right)$ to $H^{1}\left(\mathcal{O}_{Y_{2,0}}(m D)\right)$ is injective. Recursion on $m$ implies that for any $n>0$ :

- $\mathbb{C}=H^{0}\left(\mathcal{O}_{Y_{2,0}}(D)\right)=H^{0}\left(\mathcal{O}_{Y_{2,0}}(n D)\right)$, and

- the natural morphism from $H^{1}\left(\mathcal{O}_{Y_{2,0}}(D)\right)$ to $H^{1}\left(\mathcal{O}_{Y_{2,0}}(n D)\right)$ is injective.

Denote by $\mathcal{L}_{t}$ the first order infinitesimal deformation of $\mathcal{O}_{Y_{2,0}}(D)$ (along the family $\pi$ ). By standard deformation theory, we have the following commutative diagram for any $n>0$ :

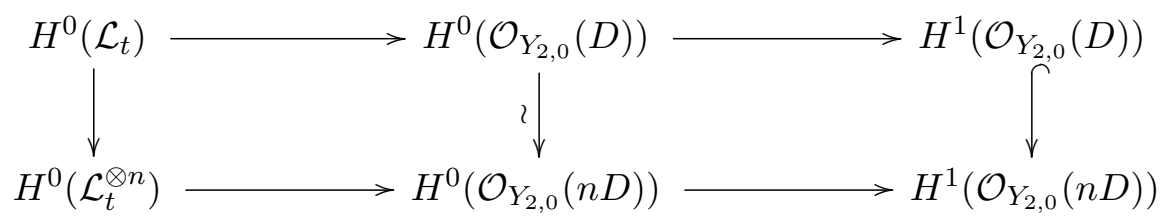

Note that there exists a first order infinitesimal deformation of $n D$ (respectively, $D$ ) if and only if the corresponding section in $H^{0}\left(\mathcal{O}_{Y_{2,0}}(n D)\right)$ (respectively, $\left.H^{0}\left(\mathcal{O}_{Y_{2,0}}(D)\right)\right)$ comes from a section of $H^{0}\left(\mathcal{L}_{t}^{\otimes n}\right)$ (respectively, $H^{0}\left(\mathcal{L}_{t}\right)$ ). Using the diagram, we then conclude that since $D$ does not deform as an effective Cartier divisor up to first order, neither does $n D$ for any $n>0$. As explained above, we then conclude that $\operatorname{Obs}_{D, \text { loc }}^{\pi}=\mathbb{C}$.

We will now observe that the difference between $\operatorname{Obs}_{D}^{\pi}$ and $\operatorname{Obs}_{D \text {,loc }}^{\pi}$ arises from 1-cocycles of the structure sheaf of $X$, after removing a codimension 2 subvariety. More precisely, let $T \subseteq D$ be a proper, closed subvariety (by proper we mean that $\operatorname{dim}_{x} T<\operatorname{dim}_{x} D$ for all $x \in T$ ) of $D$. Let

$$
\Phi_{(T \subseteq D)}: H^{1}\left(\mathcal{O}_{X \backslash T}\right) \longrightarrow H_{D}^{2}\left(\mathcal{O}_{X}\right)
$$

be the composition of homomorphisms

$$
H^{1}\left(\mathcal{O}_{X \backslash T}\right) \longrightarrow H^{1}\left(\mathcal{O}_{X \backslash D}\right) \longrightarrow H_{D}^{2}\left(\mathcal{O}_{X}\right),
$$

where the first morphism is natural and the second morphism arises from the short exact sequence $(2.2)$ (note that, $H^{1}\left(\mathcal{O}_{X \backslash D}\right) \cong H^{1}\left(j_{*}\left(\mathcal{O}_{X \backslash D}\right)\right)$ as $j$ is an affine morphism). From the exactness of the sequence

$$
H^{1}\left(\mathcal{O}_{X \backslash D}\right) \longrightarrow H_{D}^{2}\left(\mathcal{O}_{X}\right) \longrightarrow H^{2}\left(\mathcal{O}_{X}\right)
$$

it follows that the cokernel of the morphism from $H^{1}\left(\mathcal{O}_{X \backslash D}\right)$ to $H_{D}^{2}\left(\mathcal{O}_{X}\right)$ is naturally contained in $H^{2}\left(\mathcal{O}_{X}\right)$. This induces a natural map:

$$
\eta_{T}: \operatorname{coker}\left(\Phi_{(T \subseteq D)}\right) \longrightarrow H^{2}\left(\mathcal{O}_{X}\right) .
$$

Theorem 4.2. There exists a non-trivial, proper, closed subvariety $T \subseteq D$, for which the image of $\operatorname{Obs}_{D, \text { loc }}^{\pi}$ in $\operatorname{coker}\left(\Phi_{(T \subseteq D)}\right)$ maps isomorphically to $\operatorname{Obs}_{D}^{\pi}$, under the natural morphism $\eta_{T}$ mentioned above. 
Proof. Let $E$ be an effective divisor on $X$. Set $T:=D$.E. We then have the following commutative diagram of exact sequences where both the horizontal rows and the vertical columns are exact:

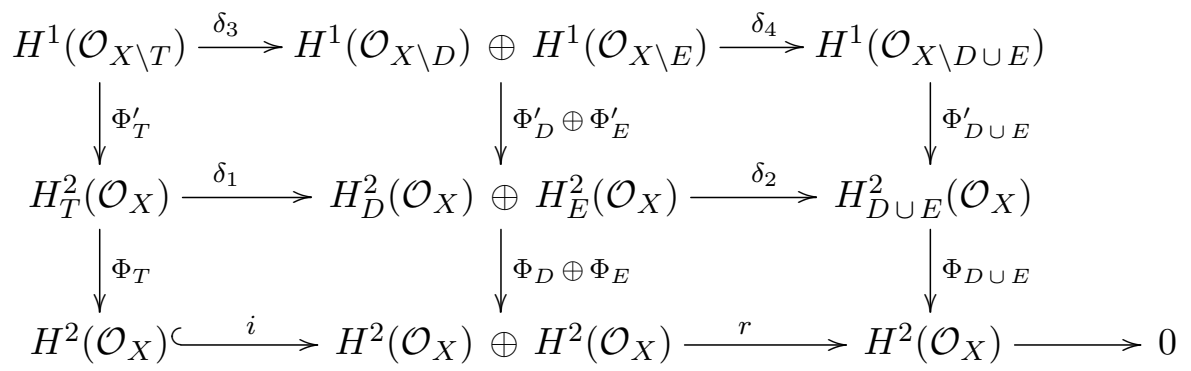

In (4.3), the top two rows are the Mayer-Vietoris sequences (see [8, Ex. III.2.4]), $i(a)=a \oplus a$, $r(a \oplus b)=a-b$ and $\Phi_{(-)}, \Phi_{(-)}^{\prime}$ are natural morphisms arising from Lemma 2.1. We want to show that in the case when $E$ is sufficiently ample, if there exists a non-zero element $\xi$ in the image of $\pi_{D} \circ \mathrm{KS}$ which maps to zero under $\Phi_{D}$, then $\xi$ lies in the image of $\Phi_{(T \subseteq D)}=\Phi_{D}^{\prime} \circ \delta_{3}$.

As $E$ is sufficiently ample (in particular, $\mathcal{O}_{X}(D)(E)$ and $\mathcal{O}_{X}(E)$ are very ample), we have $X \backslash E$ and $X \backslash(D \cup E)$ are affine. This implies

$$
H^{1}\left(\mathcal{O}_{X \backslash E}\right)=0=H^{1}\left(\mathcal{O}_{X \backslash(D \cup E)}\right) .
$$

Hence, $\Phi_{E}$ and $\Phi_{D \cup E}$ are injective morphisms. This implies that for any $\xi \in \operatorname{ker}\left(\Phi_{D}\right)$, we have $\delta_{2}(\xi \oplus 0)=0$. Hence, by the exactness of the middle horizontal row of $(4.3)$, there exists $\xi_{1}^{\prime} \in H_{T}^{2}\left(\mathcal{O}_{X}\right)$ such that $\delta_{1}\left(\xi_{1}^{\prime}\right)=\xi \oplus 0$. By the injectivity of $i$, note that $\Phi_{T}\left(\xi_{1}^{\prime}\right)=0$. Then, by the exactness of the first vertical column of (4.3), there exists $\xi^{\prime} \in H^{1}\left(\mathcal{O}_{X \backslash T}\right)$ such that $\Phi_{T}^{\prime}\left(\xi^{\prime}\right)=\xi_{1}^{\prime}$. By the commutativity of the upper left hand square in (4.3), we have that

$$
\xi \oplus 0=\delta_{1} \circ \Phi_{T}^{\prime}\left(\xi^{\prime}\right)=\left(\Phi_{D}^{\prime} \oplus \Phi_{E}^{\prime}\right) \circ \delta_{3}\left(\xi^{\prime}\right) .
$$

In particular, $\Phi_{D}^{\prime} \circ \delta_{3}\left(\xi^{\prime}\right)=\xi$. Note that, for $T=D . E$, we have (by definition) $\Phi_{D}^{\prime} \circ \delta_{3}=\Phi_{T \subset D}$. This means, for any $\xi \in \operatorname{ker}\left(\Phi_{D}\right)$, there exists $\xi^{\prime} \in H^{1}\left(\mathcal{O}_{X \backslash T}\right)$ such that $\Phi_{T \subseteq D}\left(\xi^{\prime}\right)=\xi$. This implies that the image of $\operatorname{Obs}_{D \text {, loc }}^{\pi}$ in $\operatorname{coker}\left(\Phi_{(T \subseteq D)}\right)$ maps isomorphically to $\operatorname{Obs}_{D}^{\pi}$, under the natural morphism $\eta_{T}$ mentioned above. This proves the theorem.

The above theorem motivates the following definition:

Definition 4.3. Given a proper, closed subvariety $T \subseteq D$, we say that $D$ is $T$-semi-regular (along $B$ ) if the conclusion of Theorem 4.2 holds for such $T$ i.e., the image of $\operatorname{Obs}_{D \text {,loc }}^{\pi}$ in $\operatorname{coker}\left(\Phi_{(T \subseteq D)}\right)$ maps isomorphically to $\operatorname{Obs}_{D}^{\pi}$, under the natural morphism $\eta_{T}$ as in (4.2).

The question we now want to study is:

Question: Given an effective divisor $D$ in $X$, what are all the possible choices of proper closed subvarieties $T \subseteq D$ such that $D$ is $T$-semi-regular?

4.2. Comparison with the geometric obstruction. Let $\pi$ be the family of smooth, projective varieties described above and $D \subseteq X$ be an effective divisor. Let

$$
\mathbf{O}_{D}^{\pi}:=\operatorname{Im}\left(\mathrm{Ob}_{D}\right) \circ \mathrm{KS} \subseteq H^{1}\left(\mathcal{N}_{D \mid X}\right)
$$

be the obstruction space for deforming $D$ as an effective Cartier divisor. Recall that the morphism $\pi_{D}^{\prime}$ from $H^{1}\left(\mathcal{N}_{D \mid X}\right)$ to $H_{D}^{2}\left(\mathcal{O}_{X}\right)$ induced by the contraction morphism $\lrcorner\{D\}^{\prime}$ as defined in $\S 2.3$. Let

$$
\operatorname{Tors}_{D}:=\operatorname{ker}\left(\mathbf{O}_{D}^{\pi} \stackrel{\pi_{D}^{\prime}}{\longrightarrow} H_{D}^{2}\left(\mathcal{O}_{X}\right)\right)
$$


be the kernel. By Theorem 3.3, Tors ${ }_{D}$ encodes information on infinitesimal deformations of $X$ such that $D$ does not deform as an effective Cartier divisor, but some multiple of $D$ does deform. By the first isomorphism theorem, the morphism $\pi_{D}^{\prime}$ induces an isomorphism from $\mathbf{O}_{D}^{\pi} /$ Tors $_{D}$ to $\operatorname{Obs}_{D, \text { loc }}^{\pi}$. Then, the following composed morphism is surjective:

$$
\mathbf{O}_{D}^{\pi} / \operatorname{Tors}_{D} \stackrel{\pi_{D}^{\prime}}{\sim} \operatorname{Obs}_{D, \text { loc }}^{\pi} \stackrel{\Phi_{D}}{\longrightarrow} \operatorname{Obs}_{D}^{\pi} .
$$

We say that $D$ is effective Lefschetz if this composed morphism is an isomorphism (this means that the fundamental class of $D$ deforms as a Hodge class if and only if a multiple of $D$ deforms as an effective Cartier divisor). We now give a partial answer to the question posed above.

Theorem 4.4. Let $D \subseteq X$ be an effective divisor. The following two hold:

(1) $D$ is $T$-semi-regular for every proper subvariety $T \subseteq D$ if and only if $D$ is effective Lefschetz,

(2) let $\mathcal{L}$ be an invertible sheaf on $\mathcal{X}$ and $E \in\left|\mathcal{L}_{o}\right|$ an effective divisor intersecting $D$ transversally, such that the union $D \cup E$ is effective Lefschetz. Then, $D$ is T-semiregular, where $T$ is the intersection product D.E.

Proof. (1) Suppose that $D$ is $T$-semi-regular for every proper closed subvariety $T \subseteq D$, in particular, in the case $T=\emptyset$. If $T=\emptyset$, then $H^{1}\left(\mathcal{O}_{X \backslash T}\right)=H^{1}\left(\mathcal{O}_{X}\right)$ and the morphism $\Phi_{(T \subseteq D)}$ coincides with the composition:

$$
H^{1}\left(\mathcal{O}_{X}\right) \longrightarrow H^{1}\left(\mathcal{O}_{X \backslash D}\right) \longrightarrow H_{D}^{2}\left(\mathcal{O}_{X}\right)
$$

which is the zero map, as this is an exact sequence (see Lemma 2.1). Hence, for $T=\emptyset$, we have $\operatorname{coker}\left(\Phi_{(T \subseteq D)}\right) \cong H_{D}^{2}\left(\mathcal{O}_{X}\right)$ and the morphism $\eta_{T}$ from $\operatorname{coker}\left(\Phi_{(T \subseteq D)}\right)$ to $H^{2}\left(\mathcal{O}_{X}\right)$, as in (4.2), coincides with $\Phi_{D}: H_{D}^{2}\left(\mathcal{O}_{X}\right) \longrightarrow H^{2}\left(\mathcal{O}_{X}\right)$. Then, by the definition of $T$-semi-regularity for $T=\emptyset$, we have that $\Phi_{D}$ maps $\operatorname{Obs}_{D \text {, loc }}^{\pi}$ isomorphically to $\operatorname{Obs}_{D}^{\pi}$, i.e., $D$ is effective Lefschetz.

Conversely, suppose that $D$ is effective Lefschetz. Note that, for any proper closed subset $T \subseteq D$, the morphism $\Phi_{D}$ factors as:

$$
H_{D}^{2}\left(\mathcal{O}_{X}\right) \longrightarrow \operatorname{coker}\left(\Phi_{(T \subseteq D)}\right) \stackrel{\eta_{T}}{\longrightarrow} H^{2}\left(\mathcal{O}_{X}\right) .
$$

By assumption, $\Phi_{D}$ maps $\operatorname{Obs}_{D \text {,loc }}^{\pi}$ isomorphically to $\operatorname{Obs}_{D}^{\pi}$. Hence, the image of $\operatorname{Obs}_{D \text {,loc }}^{\pi}$ in $\operatorname{coker}\left(\Phi_{(T \subseteq D)}\right)$ maps isomorphically to $\operatorname{Obs}_{D}^{\pi}$, under the morphism $\eta_{T}$. Therefore, $D$ is $T$-semiregular for any proper closed subset $T \subseteq D$. This proves (1).

(2) Consider the commutative diagram (4.3) of short exact sequences. Let

$$
\delta_{2}^{\prime}: \mathcal{H}_{D}^{1}\left(\mathcal{O}_{X}\right) \oplus \mathcal{H}_{E}^{1}\left(\mathcal{O}_{X}\right) \longrightarrow \mathcal{H}_{D \cup E}^{1}\left(\mathcal{O}_{X}\right)
$$

the natural morphism. Using the Leibniz rule, observe that

$$
\left.\left.\delta_{2}^{\prime} \circ(\lrcorner\{D\} \oplus\right\lrcorner\{E\}\right): \mathcal{T}_{X} \longrightarrow \mathcal{H}_{D \cup E}^{1}\left(\mathcal{O}_{X}\right)
$$

coincides with $\lrcorner\{D \cup E\}$. Taking cohomology, this implies $\delta_{2} \circ\left(\pi_{E} \oplus \pi_{D}\right)=\pi_{E \cup D}$. As $E \in\left|\mathcal{L}_{o}\right|$, we have $\Phi_{E}\left(\mathrm{Obs}_{E \text {,loc }}^{\pi}\right)=0$ (as there is no obstruction to the deformation of the invertible sheaf $\mathcal{O}_{X}(E)$ along $B$ ). Then, by the commutativity of the lower right hand square of (4.3), we have for any $t \in T_{o} B$,

$\Phi_{D} \circ \pi_{D} \circ \mathrm{KS}(t)=0 \Leftrightarrow\left(\Phi_{D} \oplus \Phi_{E}\right) \circ\left(\pi_{D} \oplus \pi_{E}\right) \circ \mathrm{KS}(t)=0 \oplus 0 \Leftrightarrow \Phi_{D \cup E} \circ \pi_{D \cup E} \circ \mathrm{KS}(t)=0$.

Since $D \cup E$ is effective Lefschetz, the restriction of $\Phi_{D \cup E}$ to $\operatorname{Obs}_{D \cup E \text {, loc }}^{\pi}$ is injective. This implies that for any $t \in T_{o} B$, we have $\Phi_{D} \circ \pi_{D} \circ \mathrm{KS}(t)=0$ if and only if

$$
\delta_{2}\left(\left(\pi_{D} \oplus \pi_{E}\right) \circ \mathrm{KS}(t)\right)=\pi_{D \cup E} \circ \mathrm{KS}(t)=0 .
$$


By the exactness of the middle row of (4.3), this implies for any $t \in T_{o} B, \Phi_{D} \circ \pi_{D} \circ \operatorname{KS}(t)=0$ if and only if there exists $\xi_{t} \in H_{T}^{2}\left(\mathcal{O}_{X}\right)$ (depending on $t$ ) such that $\left(\pi_{D} \oplus \pi_{E}\right) \circ \operatorname{KS}(t)=\delta_{1}\left(\xi_{t}\right)$. By the injectivity of the morphism $i$, this is equivalent to $\Phi_{T}\left(\xi_{t}\right)=0$. By the exactness of the first vertical column, we conclude that for any $t \in T_{o} B, \Phi_{D} \circ \pi_{D} \circ \mathrm{KS}(t)=0$ if and only if there exists $\xi_{t}^{\prime} \in H^{1}\left(\mathcal{O}_{X \backslash T}\right)$ such that $\Phi_{D}^{\prime} \circ \delta_{3}\left(\xi_{t}^{\prime}\right)=\pi_{D} \circ \mathrm{KS}(t)$, i.e., $\operatorname{ker}\left(\Phi_{D}\right) \cap \operatorname{Im}\left(\pi_{D} \circ \mathrm{KS}\right)=\operatorname{Im}\left(\Phi_{D}^{\prime} \circ \delta_{3}\right)$. Note that, $\Phi_{D}^{\prime} \circ \delta_{3}=\Phi_{(T \subseteq D)}$. This implies that the image of $\operatorname{Obs}_{D \text {,loc }}^{\pi}$ in $\operatorname{coker}\left(\Phi_{(T \subseteq D)}\right)$ maps isomorphically to $\operatorname{Obs}_{D}^{\pi}$, under the morphism $\eta_{T}$, i.e., $D$ is $T$-semi-regular. This proves (2).

Corollary 4.5. The following are equivalent:

(1) $D$ is saturated and $T$-semi-regular for every proper subvariety $T$ of $D$

(2) for any $t \in T_{o} B, D$ deforms as an effective Cartier divisor of the first order deformation $X_{t}$ of $X$ along $t$, if and only if the associated cohomology class $[D]$ deforms as a Hodge class in the sense that $[D] \cup \mathrm{KS}(t)=0$.

Proof. (1) $\Rightarrow(2)$ : Suppose that $D$ is saturated and $T$-semi-regular for every proper subvariety $T \subseteq D$. This implies that $\operatorname{Tors}_{D}=0$. Moreover, by Theorem 4.4, we have $D$ is effective Lefschetz. This implies that the natural morphism

$$
\mathbf{O}_{D}^{\pi} \longrightarrow \operatorname{Obs}_{D}^{\pi}
$$

is injective, which is a simple reformulation of statement (2).

$(2) \Rightarrow(1)$ : Statement (2) implies that the natural morphism from $\mathbf{O}_{D}^{\pi}$ to $\operatorname{Obs}_{D}^{\pi}$ is injective. This implies that $\operatorname{Tors}_{D}=0$ and $D$ is effective Lefschetz. Using Corollary 3.5, this implies $D$ is saturated. Then (1) follows immediately from Theorem 4.4. This proves the corollary.

\section{Applications}

In $\S 4$ above, we observed that every divisor $D$ of a smooth, projective variety $X$ is $T$-semiregular for some closed subvariety $T$ of $D$, which means that the obstruction to the first order deformation of the cohomology class of $D$ as a Hodge class lies in the cokernel of a natural morphism from $H^{1}\left(\mathcal{O}_{X \backslash T}\right)$ to $H_{D}^{2}\left(\mathcal{O}_{X}\right)$. In this section, we wish to study the variation of $T$ semi-regularity in families. We prove the existence of relative $T$-semi-regularity in Theorem 5.3. We use this to to re-interpret classical questions in deformation theory, in terms of $T$-semiregularity (see Theorems 5.3, 5.4).

5.1. Jumping locus of linear system. We use notations as in $\S 2$. Let

$$
\pi: \mathcal{X} \longrightarrow B
$$

be a flat family of smooth projective varieties with a fixed base point $o \in B$. Let $\mathcal{X}_{b}:=\pi^{-1}(b)$ be the inverse image for any $b \in B$, and define $X:=\mathcal{X}_{o}=\pi^{-1}(o)$. Fix a relative polarization $H$ on $\mathcal{X}$. Let $\mathcal{L}$ be an invertible sheaf on $\mathcal{X}$ such that $h^{0}\left(\mathcal{L}_{b}\right) \geq 1$ for all $b \in B$, where $\mathcal{L}_{b}:=\left.\mathcal{L}\right|_{\mathcal{X}_{b}}$. The goal of this subsection is to give a necessary and sufficient condition under which every divisor $D \in\left|\mathcal{L}_{o}\right|$ lifts to an effective Cartier divisor in any first-order deformation $X_{t}$ of $X$ along $B$ (Theorem 5.3).

Denote by $\mathcal{P} i c_{\mathcal{X} / B}$ the relative Picard functor associated to $\pi$ (see [2, Chapter 8, Definition 5]). Recall that $\mathcal{P} i c_{\mathcal{X} / B}$ is representable by a $B$-scheme ( $\left[2, \mathrm{n}^{o} 232\right.$, Theorem 3.1]), say Pic $\mathcal{X} / B$. Therefore, the invertible sheaf $\mathcal{L}$ on $\mathcal{X}$ induces a canonical $B$-morphism

$$
f_{\mathcal{L}}: B \longrightarrow \operatorname{Pic}_{\mathcal{X} / B}
$$

such that the composition

$$
B \stackrel{f_{\mathcal{L}}}{\longrightarrow} \operatorname{Pic}_{\mathcal{X} / B} \longrightarrow B
$$


is the identity map. Denote by $\mathcal{D}_{\mathcal{X} / B}$ the relative effective Cartier divisor functor (see $[2, \mathrm{p}$. 212]). The functor $\mathcal{D i v}_{\mathcal{X} / B}$ is representable by a $B$-scheme, say $\operatorname{Div}_{\mathcal{X} / B}$. Moreover, there is a canonical $B$-morphism:

$$
\mathfrak{d}: \operatorname{Div}_{\mathcal{X} / B} \longrightarrow \operatorname{Pic}_{\mathcal{X} / B}
$$

which associates to an effective Cartier divisor $D$ of $\mathcal{X}$, the invertible sheaf $\mathcal{O}_{\mathcal{X}}(D)$ (see $[2$, p. 214]). Let

$$
\mathfrak{d}_{\mathcal{L}}: \mathcal{D}_{\mathcal{L}} \longrightarrow B
$$

be the base change of the morphism $\mathfrak{d}$ by the morphism $f_{\mathcal{L}}$. By [11, Theorem 2.1.5], there exists an unique locally closed subscheme $W \subseteq B$ containing $o$ such that

$$
\mathfrak{d}_{\mathcal{L}}^{-1}(W) \longrightarrow W
$$

is flat and a scheme morphism $W^{\prime} \longrightarrow B$ factors through $W$ if and only if the base change $W^{\prime} \times{ }_{B} W \longrightarrow W^{\prime}$ is flat with every fiber of dimension $m$, where $m=\operatorname{dim}\left|\mathcal{L}_{o}\right|$ (note that $\left.\left|\mathcal{L}_{o}\right|=\mathfrak{d}_{\mathcal{L}}^{-1}(o)\right)$. This is called the universal property of flattening stratification.

Definition 5.1. Let $Y$ be a scheme and $\mathcal{M}$ an invertible sheaf on $Y$. A global section $s$ of $H^{0}(\mathcal{M})$ is called regular if the natural morphism

$$
s: \mathcal{O}_{Y} \longrightarrow \mathcal{M}
$$

induced by multiplication by $s$, is injective.

The following lemma gives a necessary and sufficient condition for regularity of a global section of an invertible sheaf over a non-reduced scheme.

Lemma 5.2. Let $A$ be a local Artinian $\mathcal{O}_{B, o}$-algebra. Define $\mathcal{L}_{A}:=\mathcal{L} \otimes_{\mathcal{O}_{B, o}} A$, and let

$$
\eta_{o}: H^{0}\left(\mathcal{L}_{A}\right) \longrightarrow H^{0}\left(\mathcal{L}_{o}\right)
$$

be the natural morphism. Take any $s \in H^{0}\left(\mathcal{L}_{A}\right)$ such that $\eta_{o}(s) \neq 0$. Then, $s$ is a regular section.

Proof. Let $J$ be the kernel of the morphism from $\mathcal{O}_{\mathcal{X}_{A}}$ to $\mathcal{L}_{A}$ induced by multiplication by $s$. Denote by $m_{A}$ the maximal ideal of $A$. Restricting the multiplication morphism to the closed fiber, we get a multiplication morphism by $\eta_{o}(s)$. Restricting this morphism further to $J$, we get the morphism

$$
\eta_{o}(s): \frac{J}{m_{A} . J} \longrightarrow \frac{J \otimes \mathcal{L}_{\mathcal{A}}}{m_{A} . J \otimes \mathcal{L}_{\mathcal{A}}},
$$

which is simply multiplication by $\eta_{o}(s)$. By the definition of $J$, this is a zero morphism. Since $\eta_{o}(s)$ is non-zero, this means that $J /\left(m_{A} J\right)$ annihilates the section $\eta_{o}(s)$. As $\mathcal{L}_{o}$ is an invertible sheaf, this means that $J /\left(m_{A} J\right)$ must be the zero ideal. By Nakayama lemma, this implies $J=0$ i.e., $s$ is regular. This proves the lemma.

Theorem 5.3. The following two hold:

(1) Let $\mathcal{D} \subseteq \mathcal{X}$ be a family of effective divisors parameterized by $B$ i.e., for every $b \in B$, the fiber $\mathcal{D}_{b}$ is an effective divisor in $\mathcal{X}_{b}$. Then, there exists a B-flat, proper, closed subvariety $\mathcal{T}$ of codimension 2 in $\mathcal{X}$ such that $\mathcal{D}_{b}$ is $\mathcal{T}_{b}$-semi-regular for every $b \in B$.

(2) $T_{o} W=T_{o} B$ if and only if every $D \in\left|\mathcal{L}_{o}\right|$ is saturated and $T$-semi-regular for every proper closed subvariety $T \subseteq D$.

Proof. (1): Since $\pi$ is a projective morphism, there exists a relative very ample line bundle $\mathcal{H}$ over $\mathcal{X}$, embedding $\mathcal{X}$ into a projective space over $B$ and satisfying the conditions

$$
H^{1}\left(\mathcal{O}_{\mathcal{X}_{b}}\left(\mathcal{D}_{b}\right) \otimes \mathcal{H}_{b}\right)=0 \text { and } H^{1}\left(\mathcal{H}_{b}\right)=0
$$


for all $b \in B$, where $\mathcal{H}_{b}:=\left.\mathcal{H}\right|_{\mathcal{X}_{b}}$. The existence of such an invertible sheaf $\mathcal{H}$ is guaranteed by Serre's vanishing theorem. Take a general section, say $H \in H^{0}(\mathcal{H})$, and set $\mathcal{T}:=H . \mathcal{D}$. For $H$ general, $\mathcal{T}$ is $B$-flat, proper, closed subvariety of codimension 2 in $\mathcal{X}$. The proof then follows identically as the proof of Theorem 4.2 after replacing $X$ by $\mathcal{X}_{b}, E$ by $H_{b}$ and $D$ by $\mathcal{D}_{b}$ for all $b \in B$, where $H_{b}:=H \cap \mathcal{X}_{b}$. This proves (1).

(2): Let $t: \operatorname{Spec}\left(k[\epsilon] /\left(\epsilon^{2}\right)\right) \longrightarrow W$ correspond to a tangent vector $T_{o} W$ i.e., the closed point of $\operatorname{Spec}\left(k[\epsilon] /\left(\epsilon^{2}\right)\right)$ maps to $o$, under $t$. Let

$$
\mathfrak{d}_{t}: \mathcal{D}_{t} \longrightarrow \operatorname{Spec}\left(k[\epsilon] /\left(\epsilon^{2}\right)\right)
$$

be the base change of $\mathfrak{d}_{\mathcal{L}}$ under the morphism $t$. By the universal property of flattening stratification mentioned above, $t \in T_{o} W$ if and only if $\mathcal{D}_{t}$ is flat over $\operatorname{Spec}\left(k[\epsilon] /\left(\epsilon^{2}\right)\right)$. Since $\mathcal{D}_{o}:=\mathfrak{d}_{\mathcal{L}}^{-1}(o)$ is smooth (projective space), $\mathfrak{d}_{t}$ is flat if and only if it is smooth. This implies that $t \in T_{o} W$ if and only if for every local Artinian $k[\epsilon] /\left(\epsilon^{2}\right)$-algebra $A$ and a small extension of $A$

$$
0 \longrightarrow I \longrightarrow A^{\prime} \stackrel{\eta}{\rightarrow} A \longrightarrow 0
$$

the natural map $\mathcal{D}_{t}\left(\operatorname{Spec}\left(A^{\prime}\right)\right) \longrightarrow \mathcal{D}_{t}(\operatorname{Spec}(A))$ is surjective. Define

$$
\mathcal{L}_{A}:=\mathcal{L} \otimes_{\mathcal{O}_{B, o}} A \quad \text { and } \quad \mathcal{L}_{A^{\prime}}:=\mathcal{L} \otimes_{\mathcal{O}_{B, o}} A^{\prime} .
$$

Recall, elements in $\mathcal{D}_{t}\left(\operatorname{Spec}\left(A^{\prime}\right)\right)$ (respectively, $\left.\mathcal{D}_{t}(\operatorname{Spec}(A))\right)$ is in $1-1$ correspondence with regular global sections of $\mathcal{L}_{A^{\prime}}$ (respectively, $\mathcal{L}_{A}$ ) (see [16]). Recall, [8, Propositions III.12.5 and 12.10] states that the natural morphism from $H^{0}\left(\mathcal{L}_{A^{\prime}}\right)$ to $H^{0}\left(\mathcal{L}_{A}\right)$ is surjective if and only if the homomorphism

$$
\eta_{t}: H^{0}\left(\mathcal{L}_{t}\right) \longrightarrow H^{0}\left(\mathcal{L}_{o}\right)
$$

is surjective. Using Lemma 5.2, we conclude that $t \in T_{o} W$ if and only if $\eta_{t}$ is surjective. By Corollary 4.5, any $D \in\left|\mathcal{L}_{o}\right|$ is saturated and $T$-semi-regular for every proper, closed subvariety $T \subseteq D$ if and only if for every $t \in T_{o} B$, we have $s_{D} \in \operatorname{Im}\left(\eta_{t}\right)$, where $s_{D} \in H^{0}\left(\mathcal{L}_{o}\right)$ is a global section corresponding to $D$. This proves (2).

5.2. Noether-Lefschetz locus. Denote by $U_{d}$ the space parameterizing smooth, degree $d$ surfaces in $\mathbb{P}^{3}$, and let

$$
\pi: \mathcal{X} \longrightarrow U_{d}
$$

the corresponding universal family. The Noether-Lefschetz locus, denoted by $\mathrm{NL}_{d}$, parametrizes smooth, degree $d$ surfaces in $\mathbb{P}^{3}$ with Picard number at least two. Using the Lefschetz $(1,1)$ theorem, observe that any irreducible component $L$ of $\mathrm{NL}_{d}$ is locally isomorphic to the Hodge locus $\operatorname{NL}(\gamma)$ for some $\gamma \in H^{1,1}\left(\mathcal{X}_{o}, \mathbb{C}\right) \cap H^{2}\left(\mathcal{X}_{o}, \mathbb{Z}\right)$ and $o \in L$ (see $[18, \S 5.3 .3]$ ).

Denote by $Q_{d}$ the Hilbert polynomial of a smooth, degree $d$ surface in $\mathbb{P}^{3}$ and Hilb $_{P, Q_{d}}$ the flag Hilbert scheme parameterizing pairs $(C \subseteq X)$, where $C$ (respectively, $X)$ is of Hilbert polynomial $P$ (respectively, $Q_{d}$ ) (see $[15, \S 4.5]$ ). Let

$$
\mathrm{pr}_{2}: \operatorname{Hilb}_{P, Q_{d}} \longrightarrow \operatorname{Hilb}_{Q_{d}}
$$

be the natural projection.

Theorem 5.4. Let $L$ be an irreducible component of $\mathrm{NL}_{d}, o \in L$ a point and $\mathcal{X}_{o}$ the corresponding surface. Choose $C \subseteq \mathcal{X}_{o}$ such that $\overline{\mathrm{NL}([C])}=L$ (closure under Zariski topology). Then, there exists an irreducible component $W$ of $\mathrm{Hilb}_{P, Q_{d}}$ such that $\mathrm{pr}_{2}(W) \cong L$ if and only if $C$ is saturated and $T$-semi-regular for every proper, closed, subvariety $T$ of $C$.

Proof. This follows directly from Corollary 4.5. 


\section{An EXAMPLE}

In this section, we produce a family $\pi: \mathcal{X} \longrightarrow B$ along with a point $o \in B$ and an effective Cartier divisor $D \subseteq \mathcal{X}_{o}$, which does not satisfy the effective Lefschetz property i.e. there exists a first order infinitesimal deformation of $\mathcal{X}_{o}$ corresponding to a tangent vector $t \in T_{o} B$ such that $D$ does not deform as an effective Cartier divisor but the cohomology class of $D$ deforms as a Hodge class (Theorem 6.3). The example is motivated by Remark 4.1. In particular, the divisor $D$ arises from the limit of the blow-up of a fixed smooth, projective surface along a finite set of points, as the set of points converges to the base locus of a linear system.

Notation 6.1. Let $Y$ be a smooth projective surface and $\mathcal{L}$ an invertible sheaf on $Y$. Denote by $F$ the reduced base locus of $\mathcal{L}$ (this means the base locus with reduced scheme structure). Suppose $F \neq \emptyset$. Fix a point $o \in F$ such that $o$ is a reduced base point in the sense that there exists a global section $s \in H^{0}(\mathcal{L})$ such that its image $s_{o}$ under the localization morphism

$$
H^{0}(\mathcal{L}) \longrightarrow \mathcal{L}_{o}
$$

defines a non-zero element in $\left(m_{o} / m_{o}^{2}\right) \mathcal{L}_{o}$, where $m_{o}$ is the maximal ideal of $\mathcal{O}_{Y, o}$. Denote by $B$ the union of the complement $Y \backslash F$ and the point $o$. Let $D$ be the surface in $Y \times B$ which is the union of $\{(b, b) \mid b \in B\}$ and $(F \backslash\{o\}) \times B$. Denote by

$$
\bar{\pi}: \mathcal{X} \longrightarrow Y \times B
$$

the blow-up of $Y \times B$ along $D$. Let $E \subseteq \mathcal{X}$ be the exceptional divisor. Let

$$
\pi: \mathcal{X} \longrightarrow B
$$

be the composition of natural morphisms

$$
\mathcal{X} \stackrel{\bar{\pi}}{\rightarrow} Y \times B \stackrel{\mathrm{pr}_{2}}{\longrightarrow} B,
$$

and let

$$
\left.\pi\right|_{E}: E \longrightarrow B
$$

be the restriction of $\pi$ to $E$. Denote by $\mathcal{X}_{b}:=\pi^{-1}(b)$ and $E_{b}:=\left(\left.\pi\right|_{E}\right)^{-1}(b)$ for all $b \in B$.

Lemma 6.2. The above morphisms $\pi$ and $\left.\pi\right|_{E}$ are flat.

Proof. Let $\mathrm{pr}_{2}: D \longrightarrow B$ be the natural projection. For every $b \in B$,

$$
\operatorname{pr}_{2}^{-1}(b)=((F \backslash\{o\}) \times\{b\}) \cup(\{b\} \times\{b\}) .
$$

Hence, $\mathrm{pr}_{2}$ is a flat morphism (to prove this use [8, Theorem III.9.9]). By the definition of a blow-up, observe that $\mathcal{X}$ is flat over $B$, under the morphism $\pi$. The flatness of $\left.\pi\right|_{E}$ follows directly from the description of the exceptional divisor of a blow-up along a regular subscheme as given in $[13, \S 8.1 .2$, Theorem 1.19]. This proves the lemma.

Consider the following short exact sequence of sheaves on $\mathcal{X}$ :

$$
0 \longrightarrow \mathcal{O}_{\mathcal{X}}(-E) \longrightarrow \mathcal{O}_{\mathcal{X}} \longrightarrow \mathcal{O}_{E} \longrightarrow 0 \text {. }
$$

We are interested in the invertible sheaf arising from the pull-back of $\mathcal{L}$ under the natural morphism from $\mathcal{X}$ to $Y$, after twisting by the ideal sheaf of the exceptional divisor. More precisely, consider the composed morphism

$$
\pi_{1}: \mathcal{X} \stackrel{\bar{\pi}}{\rightarrow} Y \times B \stackrel{\mathrm{pr}_{1}}{\rightarrow} Y
$$

Define

$$
\mathcal{M}:=\pi_{1}^{*} \mathcal{L} \otimes \mathcal{O}_{\mathcal{X}}(-E)
$$


Tensoring (6.1) by $\pi_{1}^{*} \mathcal{L}$, we get the following short exact sequences:

$$
0 \longrightarrow \mathcal{M} \longrightarrow \pi_{1}^{*} \mathcal{L} \longrightarrow \pi_{1}^{*} \mathcal{L} \otimes \mathcal{O}_{E} \longrightarrow 0
$$

For any $b \in B$, let $\mathcal{M}_{b}:=\left.\mathcal{M}\right|_{\mathcal{X}_{b}}$. We then observe:

Theorem 6.3. Let KS : $T_{o} B \longrightarrow H^{1}\left(\mathcal{T}_{\mathcal{X}_{o}}\right)$ be the Kodaira-Spencer map associated to $\pi$. There exists $t \in T_{o} B$ and $C \in\left|\mathcal{M}_{o}\right|$ such that $\mathrm{KS}(t) \cup[C]=0$ but $C$ does not lift to an effective Cartier divisor of the corresponding first order infinitesimal deformation $\mathcal{X}_{o, t}$ of $\mathcal{X}_{o}$.

Proof. Any tangent vector $t \in T_{o} B$ corresponds to a ring morphism,

$$
\phi: \mathcal{O}_{B, o} \longrightarrow k(o)[\epsilon] /\left(\epsilon^{2}\right) .
$$

As (6.2) is a short exact sequence of $B$-flat $\mathcal{O}_{\mathcal{X}}$-modules, applying $-\otimes_{\phi} k(o)[\epsilon] /\left(\epsilon^{2}\right)$ preserves exactness:

$$
0 \longrightarrow \mathcal{M} \otimes_{\phi} \frac{k(o)[\epsilon]}{\left(\epsilon^{2}\right)} \longrightarrow \pi_{1}^{*} \mathcal{L} \otimes_{\phi} \frac{k(o)[\epsilon]}{\left(\epsilon^{2}\right)} \stackrel{\rho_{\phi}}{\longrightarrow}\left(\pi_{1}^{*} \mathcal{L} \otimes \mathcal{O}_{E}\right) \otimes_{\phi} \frac{k(o)[\epsilon]}{\left(\epsilon^{2}\right)} \longrightarrow 0 .
$$

Given any coherent sheaf $\mathcal{F}$ on $\mathcal{X}$, define the morphism

$$
\epsilon:\left(\mathcal{F} \otimes_{\phi} \frac{k(o)[\epsilon]}{\left(\epsilon^{2}\right)}\right) \otimes_{\frac{k(o)[\epsilon]}{\left(\epsilon^{2}\right)}} k(o) \longrightarrow\left(\mathcal{F} \otimes_{\phi} \frac{k(o)[\epsilon]}{\left(\epsilon^{2}\right)}\right) \otimes_{\frac{k(o)[\epsilon]}{\left(\epsilon^{2}\right)}} \frac{k(o)[\epsilon]}{\left(\epsilon^{2}\right)},
$$

which maps $s \otimes 1$ to $s \otimes \epsilon$ for $s$ a section of $\mathcal{F} \otimes_{\phi} k(o)[\epsilon] /\left(\epsilon^{2}\right)$. Applying the global section functor to the above short exact sequence, we obtain the following diagram:

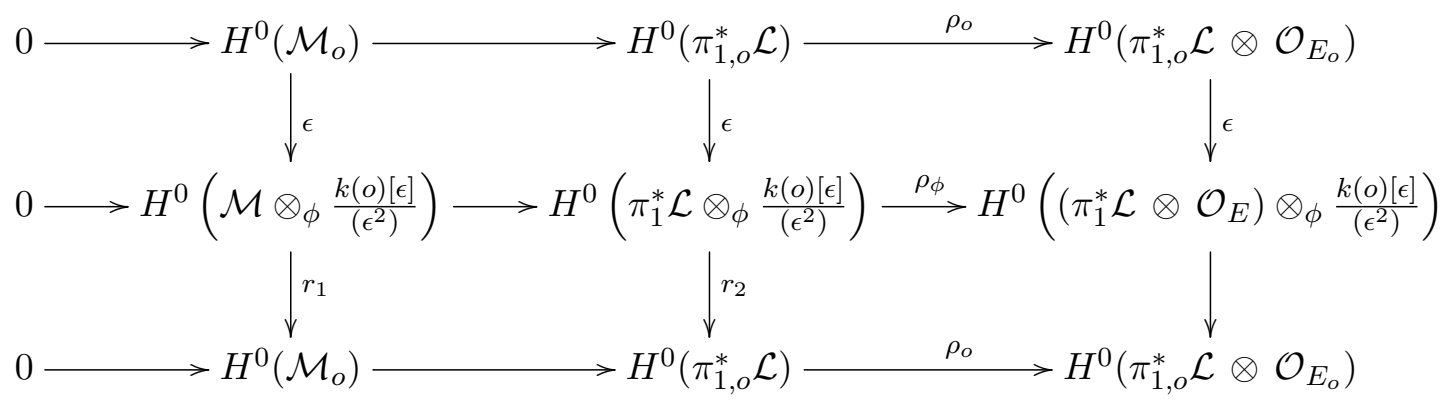

where all the rows and columns are exact and the morphisms $\epsilon$ are injective and $\pi_{1, b}: \mathcal{X}_{b} \rightarrow Y$ is the restriction of $\pi$ to $\mathcal{X}_{b}$, for any $b \in B$. Using the Zariski main theorem and the projection formula, we get

$$
\begin{aligned}
& H^{0}\left(\pi_{1, b}^{*} \mathcal{L}\right)=H^{0}\left(\pi_{1, b_{*}} \pi_{1, b}^{*} \mathcal{L}\right)=H^{0}(\mathcal{L}) \text { for all } b \in B \text { and } \\
& H^{0}\left(\pi_{1, o}^{*} \mathcal{L} \otimes \mathcal{O}_{E_{o}}\right)=H^{0}\left(\pi_{1, o_{*}}\left(\pi_{1, o}^{*} \mathcal{L} \otimes \mathcal{O}_{E_{o}}\right)\right)=H^{0}\left(\mathcal{L} \otimes \mathcal{O}_{F}\right)
\end{aligned}
$$

Since $F$ is the base locus of $\mathcal{L}$, this means the evaluation map $\rho_{o}$ is the zero map. By Grauert's upper semicontinuity theorem [8, Corollary III.12.9], the morphism $r_{2}$ is surjective. If $r_{1}$ is surjective, then the Snake lemma implies that $\rho_{\phi}$ is the zero map. Therefore, it suffices to show that $\rho_{\phi}$ is not the zero map. Indeed, this would imply that $r_{1}$ is not surjective. This directly means that there exists $C \in\left|\mathcal{M}_{o}\right|$ not contained in $\operatorname{Im} r_{1}$. In other words, $C$ does not lift to an effective Cartier divisor of the corresponding first order infinitesimal deformation $\mathcal{X}_{o, t}$ of $\mathcal{X}_{o}$. This will prove the theorem.

Since $\pi_{1}=\bar{\pi} \circ \mathrm{pr}_{1}$, the projection formula along with Zariski's main theorem implies that

$$
\begin{gathered}
H^{0}\left(\pi_{1}^{*} \mathcal{L} \otimes_{\phi} k(o)[\epsilon] /\left(\epsilon^{2}\right)\right)=H^{0}\left(\operatorname{pr}_{1}^{*} \mathcal{L} \otimes_{\phi} k(o)[\epsilon] /\left(\epsilon^{2}\right)\right) \text { and } \\
H^{0}\left(\pi_{1}^{*} \mathcal{L} \otimes \mathcal{O}_{E} \otimes_{\phi} k(o)[\epsilon] /\left(\epsilon^{2}\right)\right)=H^{0}\left(\operatorname{pr}_{1}^{*} \mathcal{L} \otimes \mathcal{O}_{D} \otimes_{\phi} k(o)[\epsilon] /\left(\epsilon^{2}\right)\right)= \\
=\bigoplus_{q \in F} H^{0}\left(\left(\operatorname{pr}_{1}^{*} \mathcal{L} \otimes \mathcal{O}_{D}\right)_{q \times o} \otimes_{\phi} k(p)[\epsilon] /\left(\epsilon^{2}\right)\right) .
\end{gathered}
$$


By assumption, there exist $s \in H^{0}(\mathcal{L}), f_{s} \in m_{o} \backslash m_{o}^{2}$ and $g_{s} \in \mathcal{L}_{o}$ (the localization of $\mathcal{L}$ at $o$ ) such that $s_{o}=f_{s} g_{s}$ and $g_{s} \notin m_{o} \mathcal{L}_{o}$; here $s_{o}$ is the image of $s$ under the localization morphism $H^{0}(\mathcal{L}) \longrightarrow \mathcal{L}_{o}$. Since $f_{s} \in m_{o} \backslash m_{o}^{2}$ and $Y$ is a smooth surface, we can choose a regular sequence $\left(f_{s}, f_{1}\right)$ generating the maximal ideal $m_{o}$. Define,

$$
\phi: \mathcal{O}_{B, o} \longrightarrow k(o)[\epsilon] /\left(\epsilon^{2}\right)
$$

the ring morphism by $1 \longmapsto 1, f_{s} \longmapsto \epsilon$ and $f_{1} \longmapsto 0$. Then, $s$ defines a non-zero element

$$
s \otimes 1 \in H^{0}\left(\operatorname{pr}_{1}^{*} \mathcal{L} \otimes_{\phi} k(o)[\epsilon] /\left(\epsilon^{2}\right)\right)
$$

and the image of $s \otimes 1$ under the natural localization morphism

$$
\rho(\phi)_{o}^{\prime}: H^{0}\left(\operatorname{pr}_{1}^{*} \mathcal{L} \otimes_{\phi} k(o)[\epsilon] /\left(\epsilon^{2}\right)\right) \longrightarrow H^{0}\left(\mathcal{L}_{o} \otimes_{\phi} k(o)[\epsilon] /\left(\epsilon^{2}\right)\right)
$$

is non-zero (use $\left.\left(\operatorname{pr}_{1}^{*} \mathcal{L}\right)_{(o, o)}=\left(\mathcal{L} \otimes_{k} \mathcal{O}_{B}\right)_{(o, o)}=\mathcal{L}_{o} \otimes_{k} \mathcal{O}_{B, o}\right)$.

Recall, the composition $\Delta \hookrightarrow Y \times Y \stackrel{\mathrm{pr}_{1}}{\longrightarrow} Y$ is an isomorphism. Hence,

$$
\operatorname{pr}_{1}^{\#}: \mathcal{O}_{Y, o} \stackrel{\sim}{\longrightarrow} \mathcal{O}_{\Delta, o \times o} .
$$

Since the only irreducible component of $D$ containing $o \times o$ is $\Delta$, we have

$$
\left(\operatorname{pr}_{1}^{*} \mathcal{L} \otimes \mathcal{O}_{D}\right)_{o \times o} \otimes_{\phi} k(o)[\epsilon] /\left(\epsilon^{2}\right) \cong \mathcal{L}_{o} \otimes_{\operatorname{pr}_{1}^{\#}} \mathcal{O}_{\Delta, o \times o} \otimes_{\phi} k(o)[\epsilon] /\left(\epsilon^{2}\right) \cong \mathcal{L}_{o} \otimes_{\phi} k(o)[\epsilon] /\left(\epsilon^{2}\right)
$$

Write the evaluation map $\rho_{\phi}=\bigoplus_{q \in F} \rho_{\phi, q}$, where $\rho_{\phi, q}$ is the restriction of the evaluation map to $q$. Then, $\rho_{\phi, o}$ coincides with the morphism

$$
H^{0}\left(\pi_{1}^{*} \mathcal{L} \otimes_{\phi} k(o)[\epsilon] /\left(\epsilon^{2}\right)\right)=H^{0}\left(\operatorname{pr}_{1}^{*} \mathcal{L} \otimes_{\phi} k(o)[\epsilon] /\left(\epsilon^{2}\right)\right) \stackrel{\rho(\phi)_{o}^{\prime}}{\longrightarrow} H^{0}\left(\mathcal{L}_{o} \otimes_{\phi} k(o)[\epsilon] /\left(\epsilon^{2}\right)\right) .
$$

Since $\rho(\phi)_{o}^{\prime}$ is non-zero, so is $\rho_{\phi}$. This completes the proof of the theorem.

\section{ACKNOWLEDGEMENTS}

The comments of the referee helped us to reformulate the results of $\S 4$ in the previous version, in terms of the local obstruction theory and improve the overall exposition of the article. We are grateful for his/her feedbacks and suggestions. The first-named author acknowledges support of a J. C. Bose Fellowship. The second author is currently supported by ERCEA Consolidator Grant 615655-NMST and also by the Basque Government through the BERC $2014-2017$ program and by Spanish Ministry of Economy and Competitiveness MINECO: BCAM Severo Ochoa excellence accreditation SEV-2013 - 0323.

\section{REFERENCES}

[1] S. Bloch. Semi-regularity and de-Rham cohomology. Inventiones Math., 17:51-66, 1972.

[2] S. Bosch, W. Lütkebohmert, and M. Raynaud. Néron models, volume 21. Springer Science \& Business Media, 2012 .

[3] M. P. Brodmann and R. Y. Sharp. Local cohomology: an algebraic introduction with geometric applications, volume 136. Cambridge university press, 2012.

[4] W. Bruns and H. J. Herzog. Cohen-Macaulay rings. Cambridge University Press, 1998.

[5] A. Dan and I. Kaur. Semi-regular varieties and variational Hodge conjecture. Comptes Rendus Mathematique, 354(3):297-300, 2016.

[6] A Grothendieck. Fondements de la géométrie algébrique (extraits du Séminaire Bourbaki, 1957-1962). 1962.

[7] R. Hartshorne. On the De Rham cohomology of algebraic varieties. Inst. Hautes Études Sci. Publ. Math., 45:5-99, 1975.

[8] R. Hartshorne. Algebraic Geometry. Graduate text in Mathematics-52. Springer-Verlag, 1977.

[9] R. Hartshorne. Deformation Theory. Graduate text in Mathematics. Springer-Verlag, 2010.

[10] R. Hartshorne and A. Grothendieck. Local cohomology: a seminar, volume 41. Springer-Verlag, 1967.

[11] D. Huybrechts and M. Lehn. The geometry of moduli spaces of sheaves. Springer, 2010. 
[12] K. Kodaira and D. C. Spencer. A theorem of completeness of characteristic systems of complete continuous systems. Amer. J. Math., 81(2):477-500, 1959.

[13] Q. Liu. Algebraic geometry and arithmetic curves, volume 6. Oxford University Press, Oxford, 2002.

[14] M. Reid. Minimal models of canonical 3-folds. In Algebraic varieties and analytic varieties, pages 131-180. Mathematical Society of Japan, 1983.

[15] E. Sernesi. Deformaions of Algebraic Schemes. Grundlehren der Mathematischen Wissenschaften-334. Springer-Verlag, 2006.

[16] The Stacks project authors. The stacks project. https://stacks.math.columbia.edu/tag/0C4S, 2018.

[17] C. Voisin. Hodge Theory and Complex Algebraic Geometry-I. Cambridge studies in advanced mathematics-76. Cambridge University Press, 2002.

[18] C. Voisin. Hodge Theory and Complex Algebraic Geometry-II. Cambridge studies in advanced mathematics77. Cambridge University Press, 2003.

School of Mathematics, Tata Institute of Fundamental Research, Homi Bhabha Road, Mumbai 400005, INDIA

Email address: indranil@math.tifr.res.in

BCAM - Basque Centre for Applied Mathematics, Alameda de Mazarredo 14, 48009 Bilbao, SPAIN

Email address: adan@bcamath.org 